\title{
EMERGENCE OF GRANULAR-SIZED MAGNETIC BUBBLES THROUGH THE SOLAR ATMOSPHERE. I. SPECTROPOLARIMETRIC OBSERVATIONS AND SIMULATIONS
}

\author{
Ada Ortiz ${ }^{1}$, Luis R. Bellot Rubio ${ }^{2}$, Viggo H. Hansteen ${ }^{1}$, \\ JaIme DE La CRUZ RodRÍGuez ${ }^{3,4}$, AND LuC RoupPe VAN DER VOORT ${ }^{1}$ \\ ${ }^{1}$ Institute of Theoretical Astrophysics, University of Oslo, P.O. Box 1029 Blindern, N-0315 Oslo, Norway; ada @astro.uio.no \\ 2 Instituto de Astrofísica de Andalucía (CSIC), Apdo. 3040, E-18080 Granada, Spain \\ ${ }^{3}$ Department of Physics and Astronomy, Uppsala University, Box 516, SE-75120 Uppsala, Sweden \\ ${ }^{4}$ Department of Astronomy, Institute for Solar Physics, Stockholm University, AlbaNova University Centre, SE-106 91 Stockholm, Sweden \\ Received 2013 September 13; accepted 2013 December 11; published 2014 January 16
}

\begin{abstract}
We study a granular-sized magnetic flux emergence event that occurred in NOAA 11024 in 2009 July. The observations were made with the CRISP spectropolarimeter at the Swedish $1 \mathrm{~m}$ Solar Telescope achieving a spatial resolution of 0!'14. Simultaneous full Stokes observations of the two photospheric Fe I lines at $630.2 \mathrm{~nm}$ and the chromospheric Ca II $854.2 \mathrm{~nm}$ line allow us to describe in detail the emergence process across the solar atmosphere. We report here on three-dimensional (3D) semi-spherical bubble events, where instead of simple magnetic footpoints, we observe complex semi-circular feet straddling a few granules. Several phenomena occur simultaneously, namely, abnormal granulation, separation of opposite-polarity legs, and brightenings at chromospheric heights. However, the most characteristic signature in these events is the observation of a dark bubble in filtergrams taken in the wings of the Ca II $854.2 \mathrm{~nm}$ line. There is a clear coincidence between the emergence of horizontal magnetic field patches and the formation of the dark bubble. We can infer how the bubble rises through the solar atmosphere as we see it progressing from the wings to the core of Ca II $854.2 \mathrm{~nm}$. In the photosphere, the magnetic bubble shows mean upward Doppler velocities of $2 \mathrm{~km} \mathrm{~s}^{-1}$ and expands at a horizontal speed of $4 \mathrm{~km} \mathrm{~s}^{-1}$. In about 3.5 minutes it travels some $1100 \mathrm{~km}$ to reach the mid chromosphere, implying an average ascent speed of $5.2 \mathrm{~km} \mathrm{~s}^{-1}$. The maximum separation attained by the magnetic legs is 6.'6. From an inversion of the observed Stokes spectra with the SIR code, we find maximum photospheric field strengths of $480 \mathrm{G}$ and inclinations of nearly $90^{\circ}$ in the magnetic bubble interior, along with temperature deficits of up to $250 \mathrm{~K}$ at $\log \tau=-2$ and above. To aid the interpretation of the observations, we carry out 3D numerical simulations of the evolution of a horizontal, untwisted magnetic flux sheet injected in the convection zone, using the Bifrost code. The computational domain spans from the upper convection zone to the lower corona. In the modeled chromosphere, the rising flux sheet produces a large, cool, magnetized bubble. We compare this bubble with the observed ones and find excellent agreement, including similar field strengths and velocity signals in the photosphere and chromosphere, temperature deficits, ascent speeds, expansion velocities, and lifetimes.
\end{abstract}

Key words: Sun: chromosphere - Sun: magnetic fields - Sun: photosphere

Online-only material: animation, color figures

\section{INTRODUCTION}

The currently accepted picture of the active Sun portrays solar magnetic fields being created as magnetic flux tubes in the tachocline (the interface between the radiative interior and the differentially rotating outer convection zone of the Sun) because of extreme shear. Buoyancy instabilities cause the flux tubes to rise as $\Omega$-shaped loops and reach the upper convection zone, emerging into the photosphere in the form of active regions (see, e.g., Caligari et al. 1995; Moreno-Insertis \& Emonet 1996; Fisher et al. 2000). This scenario is supported by more recent simulations that include the effects of turbulent, solar-like convective flows on rising thin flux tubes (Weber et al. 2011), three-dimensional (3D) MHD flux tubes (Jouve \& Brun 2009), and buoyant magnetic loops (Nelson et al. 2011). The emergence occurs in a plethora of sizes and magnetic flux contents, stretching from the largest activity complexes, with sunspots $30,000 \mathrm{~km}$ wide and fluxes of the order of $10^{22} \mathrm{Mx}$, to pores, faculae, and the smaller ephemeral regions, with fluxes between $10^{18}$ and $10^{20} \mathrm{Mx}$ (e.g., Martin \& Harvey 1979; Hagenaar et al. 2003).

In what concerns the quiet Sun, much has been advanced in the last decade thanks to the use of full Stokes polarimetry at high spatial resolution, as provided by ground-based and spaceborne telescopes (see Moreno-Insertis 2012). Early spectropolarimetric measurements from the ground revealed that also in the quiet Sun magnetic flux is emerging continually (e.g., Lites et al. 1996; De Pontieu 2002; Martínez González et el. 2007). The Solar Optical Telescope on board the Hinode satellite (Kosugi et al. 2007) significantly extended our observational capabilities, making it possible to characterize granular-sized flux emergence events with a resolution and sensitivity never reached before (e.g., Centeno et al. 2007; Otsuji et al. 2007; Orozco Suárez et al. 2008; Martínez González \& Bellot Rubio 2009; Wang et al. 2012). Using these data, Ishikawa et al. (2008) and Ishikawa \& Tsuneta (2009) demonstrated that the emergence of magnetic flux on granular scales brings large amounts of horizontal fields to the quiet photosphere, while Bellot Rubio \& Orozco Suárez (2012) proved that the solar internetwork is indeed full of weak horizontal magnetic fields.

To characterize flux emergence events in the photosphere and above from the beginning to the end, one needs simultaneous measurements in all possible wavelength ranges, from the infrared to hard X-rays, at the highest spatial resolution. Therefore, having a complete observational picture of flux emergence has proven to be extremely difficult. 
Concerning new modeling capabilities, our knowledge has also advanced thanks to the availability of very extensive (sunspot-sized simulation boxes), very high resolution, realistic 3D numerical simulations of flux emergence in the solar atmosphere. According to Tortosa-Andreu \& Moreno-Insertis (2009), two classes of models can be distinguished. The first type includes models with boundaries from the top of the convection zone to the corona. In order to boost computing speed, they strongly simplify the thermodynamics of the plasma and ignore its interaction with the radiation field; in most cases, heat conduction is also neglected. Such simplifications limit the possibility of comparison with observations in the regions where radiative transfer processes are important (see Tortosa-Andreu \& Moreno-Insertis 2009, and references therein). A second class of models have appeared recently that solve the radiation transfer problem simultaneously with the MHD equations (Cheung et al. 2007, 2008; Martínez-Sykora et al. 2008; Tortosa-Andreu \& Moreno-Insertis 2009). These authors injected a horizontal magnetic flux tube or sheet (with different levels of field line twist) in the lower levels of the domain. Martínez-Sykora et al. (2008) used a larger box in the vertical direction reaching up to the chromosphere, transition region, and part of the corona. They found cold bubbles developing in coincidence with the arrival of the magnetized plasma to the chromosphere. Tortosa-Andreu \& Moreno-Insertis (2009) also found irregular and extended cool patches when the magnetized plasma reaches the chromosphere. They concluded that "the rise of the magnetized plasma in the low atmosphere proceeds in the form of a series of jumps with stops in between (at levels mostly between 200 and $500 \mathrm{~km}$ ), instead of in a continuous fashion." The reason given by Tortosa-Andreu \& Moreno-Insertis (2009) for the occurrence of these jumps and stops is the necessity of the plasma to pile up at a certain height and to expand sideways so the plasma $\beta$ decreases and buoyancy instabilities can develop again. Once this occurs, the magnetized plasma continues its way up.

The interaction between emerging flux and preexisting ambient fields has become a hot topic of research for both observers and modelers. When the two flux systems interact, reconnection is likely to happen, producing brightenings and surges. Therefore, observations of the emerging flux and its interaction with the preexisting field are important to understand key processes of energy release and associated phenomena in the solar atmosphere.

Signatures of energy release at different scales and heights have been identified above emerging flux regions repeatedly (for a review, see Guglielmino 2012). The work of Guglielmino et al. (2010) is a recent example of multiwavelength, multilayer studies of flux emergence events. Using observations in the visible, UV, EUV, and soft X-rays, they analyzed brightenings in the chromosphere, transition region, and corona, as well as chromospheric surges, associated with an intermediate-scale emerging flux region and ascribed them to the reconnection of the newly emerged flux and the preexisting field. Other examples of energy release in active regions are small-scale brightenings and transient emissions in the wings of the $\mathrm{H} \alpha$ line, the so-called Ellerman bombs (Georgoulis et al. 2002; Watanabe et al. 2011).

We have observed a new type of granular-sized flux emergence event by using high-resolution spectropolarimetric measurements in the photospheric Fe I $630.2 \mathrm{~nm}$ lines and the chromospheric Ca II $854.2 \mathrm{~nm}$ line. These events differ from others reported in the literature (e.g., Centeno et al. 2007; Martínez González \& Bellot Rubio 2009; Gömöry et al. 2010; Martínez González et al. 2010; Gömöry et al. 2013) in that they are not loop shaped but instead have a 3D semi-spherical shape, resembling a parachute. Because of their similarities, we name them magnetic bubbles, as opposed to magnetic loops. If magnetic loops intersect the photosphere at two points, called footpoints, our magnetic bubbles do so in half moon shaped magnetic feet or legs of opposite polarity. In Ca II $854.2 \mathrm{~nm}$ images, the bubbles appear dark; therefore, we simply refer to them as dark bubbles.

This paper presents a detailed characterization of these magnetic bubbles, including their temporal evolution, their magnetic and dynamic properties in different layers of the atmosphere, and a comparison with numerical simulations that show similar features. Paper II of the series will be devoted to an in-depth analysis of the observed Ca II $854.2 \mathrm{~nm}$ Stokes profiles, which will be inverted and compared with synthetic spectra calculated from the simulations.

We start by describing the observations and the data reduction in Section 2. In Section 3 we present two examples of emerging magnetic bubbles and the various phenomena associated with them. We also determine the velocity and magnetic structure of the bubbles from a bisector analysis and Stokes inversion of the observed polarization signals. Section 4 describes our numerical simulations and the bubbles they produce. Finally, in Section 5 we discuss our findings and compare them with earlier observational results and simulations.

\section{OBSERVATIONS AND DATA ANALYSIS}

\subsection{Observations}

We analyze observations of AR 11024 acquired on 2009 July 5 with the Swedish $1 \mathrm{~m}$ Solar Telescope (SST; Scharmer et al. 2003) and the CRisp Imaging Spectro-Polarimeter (CRISP; Scharmer et al. 2008). The seeing conditions were very favorable, and from the large volume of recorded data we select a period of 28 minutes starting at 09:48:40 UT. During this time, the FOV was centered near $\mathrm{S} 27^{\circ}, \mathrm{W} 12^{\circ}$. The observations consist of simultaneous full-Stokes measurements of the Fe I 630.15 and $630.25 \mathrm{~nm}$ lines and the Ca II $854.2 \mathrm{~nm}$ line. The spatial resolution of the observations is close to the diffraction limit of the telescope $(\lambda / D=0.14$ at $630 \mathrm{~nm})$.

CRISP is a tunable filter consisting of two etalons in telecentric mount. This type of instruments allows one to observe the entire field of view (FOV) in quasi-monochromatic light (the CRISP passband at $630 \mathrm{~nm}$ has a full width at half maximum of $65 \mathrm{~mA}$ ). Spectral lines are scanned by tuning the etalons. Each of the $\mathrm{Fe}$ I lines was sampled at 15 positions across the range $\pm 336 \mathrm{~m} \AA$ from the line center in steps of $48 \mathrm{~m} \AA$. A continuum point was observed at $+680 \mathrm{~m} \AA$. The Ca II $854.2 \mathrm{~nm}$ line was sampled at 17 positions across the range $\pm 800 \mathrm{~m} \AA$ in steps of $100 \mathrm{~m} \AA$, plus an extra point located at $+2.4 \AA$.

The polarization modulation was achieved with the help of two liquid crystal variable retarders cycling through four states. The polarization analysis was performed by a polarizing beam splitter in front of the two narrow-band cameras of CRISP. In order to reduce the noise, we recorded nine exposures per modulation state, resulting in an effective integration time of $153 \mathrm{~ms}$ per wavelength position and Stokes parameter. A full spectral scan of the two Fe I lines, the Ca II line, and the two continuum positions was completed in $61 \mathrm{~s}$. The time sequence contains 28 line scans with a cadence of $67 \mathrm{~s}$.

Here we will often refer to events observed in the wings and cores of the iron and calcium lines. To establish a rough relationship between the various wavelength positions and 
Fe I 6301.5

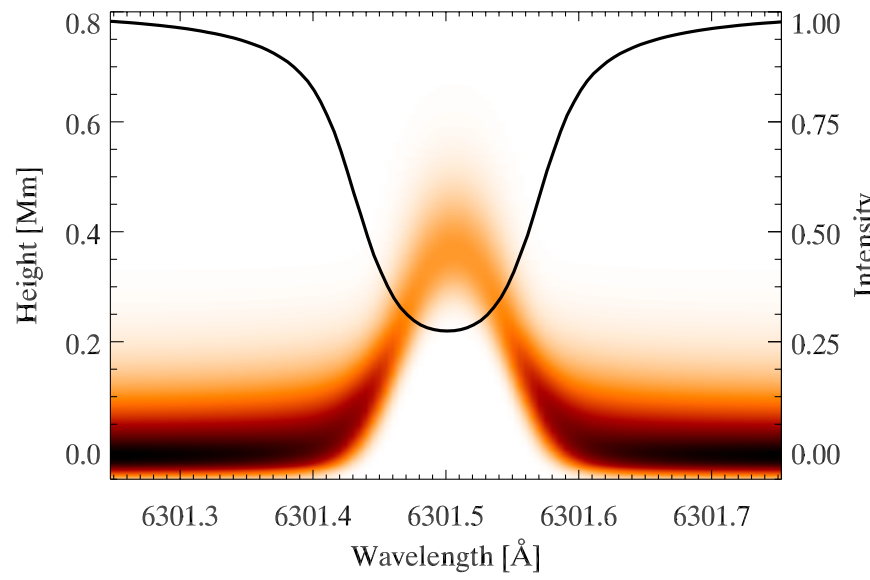

Ca II 8542

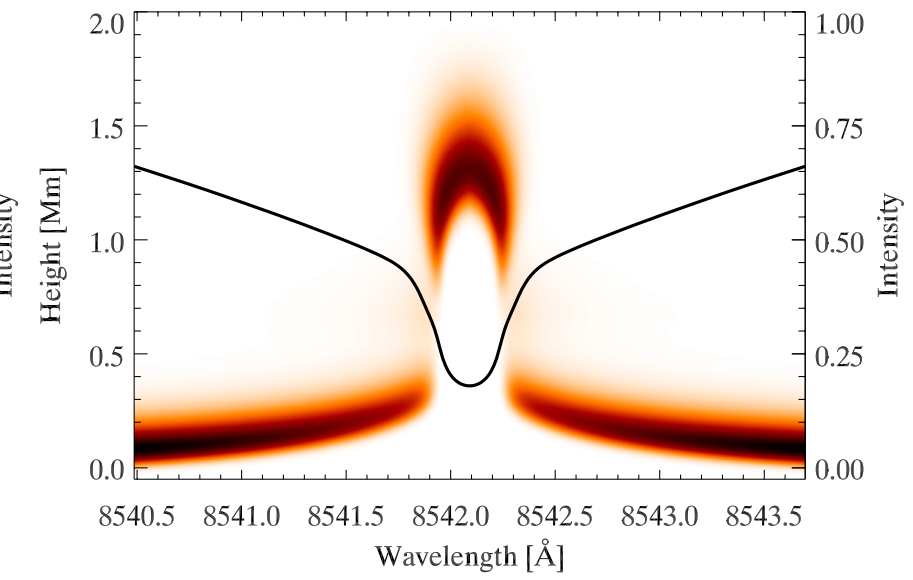

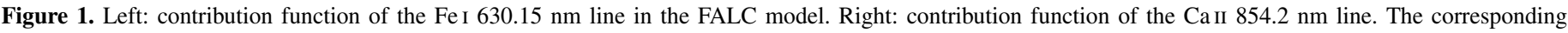

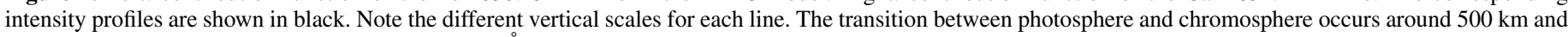
is signaled by the knees of Ca II $854.2 \mathrm{~nm}$ at $\pm 300 \mathrm{~mA}$.

(A color version of this figure is available in the online journal.)

geometrical heights, in Figure 1 we plot the contribution functions of Fe I $630.15 \mathrm{~nm}$ and Ca II $854.2 \mathrm{~nm}$ as computed in the FALC model of Fontenla et al. (1993). The $\tau=1$ layer is very corrugated in the chromosphere, especially in the presence of magnetic fields. The FALC model cannot reflect this corrugation because it is one dimensional, but it serves to illustrate the range of heights that we can expect for Ca II $854.2 \mathrm{~nm}$. Figure 1 shows that all phenomena observed in the Fe I lines happen within the photosphere (the wings referring to the low photosphere close to the continuum and the core to the upper photosphere). Events detected in the wings of the Ca II $854.2 \mathrm{~nm}$ line take place in the mid-high photosphere, while events observed in the line core occur in the mid chromosphere. For example, the Ca II filtergrams at $-0.06 \mathrm{~nm}$ from the line core probe the photosphere at a height of about $200 \mathrm{~km}$ and hence show reversed granulation. The "knees" of the intensity profile at around $\pm 300 \mathrm{~m} \AA$ sample the minimum temperature region, some $500 \mathrm{~km}$ above the continuum forming layer. The line core, representing heights of approximately $1300 \mathrm{~km}$, is purely chromospheric.

Through the use of the SST adaptive optics system and the multi-object, multi-frame blind deconvolution image restoration technique (MOMFBD; van Noort et al. 2005), we correct most of the wavefront distortions and image blurring induced by Earth's atmosphere. Precise alignment between the wideband and narrowband cameras is achieved by a separate alignment procedure involving a reference pinhole array target. The preprocessing and polarimetric calibration of the data follow the methods described in de la Cruz Rodríguez et al. (2013), and telescope-induced polarization was compensated at $630 \mathrm{~nm}$ and $854 \mathrm{~nm}$ by using the telescope models of Selbing (2005) and de la Cruz Rodríguez (2010), respectively. For each spectral line, the entire time series was aligned and derotated to a common reference, and residual rubbersheet motions were removed as described by Shine et al. (1994). Finally, the Ca II $854.2 \mathrm{~nm}$ images in each scan were aligned with respect to the Fe I 630.15 images from the same time step. To achieve high accuracy, the alignment was done using the wideband images because they show photospheric scenes in the two spectral ranges.

An inspection of the resulting Stokes profiles revealed a small amount of residual crosstalk from Stokes $I$ into $Q, U$, and $V$. We removed this contamination as follows,

$$
S(\lambda)=S_{\mathrm{obs}}(\lambda)-S_{\mathrm{offset}} \cdot I_{\mathrm{obs}}(\lambda)
$$

where $S$ represents any of the three Stokes parameters $Q, U$, and $V$, and $S_{\text {offset }}$ is the corresponding crosstalk coefficient. The various $S_{\text {offset }}$ were calculated by linear regression of $Q(\lambda), U(\lambda)$, and $V(\lambda)$ against $I(\lambda)$, for pixels with negligible polarization signals, and turn out to be of the order of $0.2 \%$. Finally, we removed small spectral gradients observed in the Stokes profiles. After calibration, the noise in Stokes $Q, U$, and $V$ is $2.5 \times 10^{-3}$, $1.5 \times 10^{-3}$, and $1.5 \times 10^{-3}$, respectively.

The observed FOV is shown in Figure 2 as seen in the red wing of the CaII $854.2 \mathrm{~nm}$ line. Magnetic bright points are prominent at this wavelength because the granulation contrast is lower than in the continuum. A train of pores (concentrations of magnetic field without a penumbra) appears embedded in the network of bright points. The observed line positions are indicated with circles in the right panel of the figure, superposed on spatially averaged quiet Sun profiles computed within the white box marked in the left panel.

AR 11024 appeared on 2009 July 4 as a pore that developed a penumbra on the same day (Schlichenmaier et al. 2010, 2012). Flux emergence was taking place intensively everywhere in the $\mathrm{AR}$, yielding numerous pores and sunspots. Here we focus on the quietest part of the FOV, which also showed many instances of flux emergence on small spatial scales. The two examples considered in this paper (cases 1 and 2) are highlighted with red boxes in the intensity and circular polarization maps of Figure 3.

\subsection{Line Parameters}

We have calculated Doppler velocities at different heights in the atmosphere from the bisectors of the Fe I $630.15 \mathrm{~nm}$ line at 10 intensity levels, from $0 \%$ (line core) to $90 \%$ (line wing near the continuum). To this end, we used the bisec_abs_lin.pro routine of the Kiepenheuer-Institut für Sonnenphysik IDL library. For the calculation of bisectors, the intensity profile is interpolated linearly. The wavelength position of the line core was obtained by means of a parabolic fit around the intensity minimum.

In order to convert bisector positions into Doppler velocities we assume that, on average, quiet Sun areas within the FOV are at rest. These areas are defined as those pixels whose 

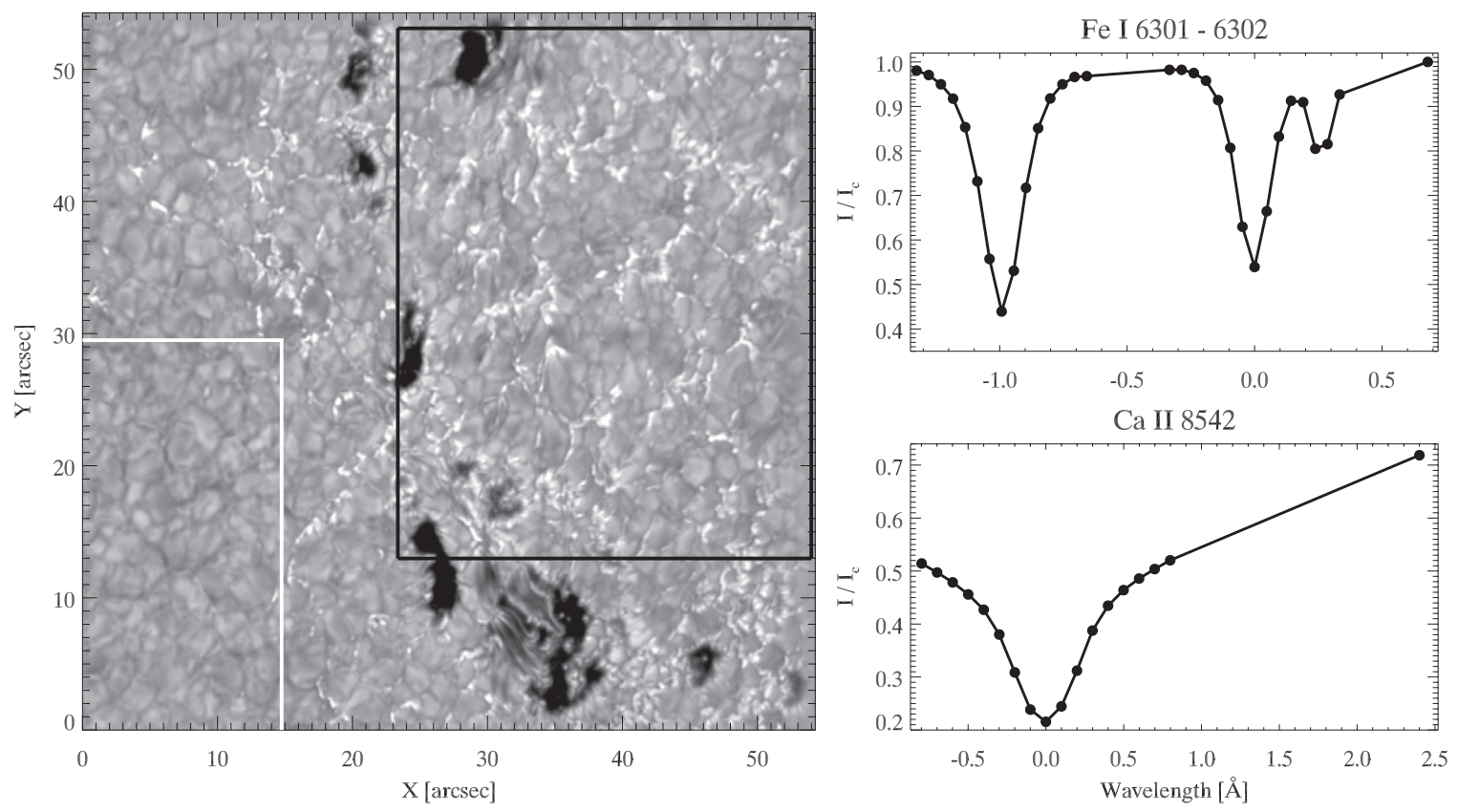

Figure 2. Left: part of active region NOAA 11024 observed $+2.4 \AA$ from the Ca II $854.2 \mathrm{~nm}$ line center. The black rectangle on the right highlights the FOV chosen for Figure 3. Right: spatially averaged quiet Sun profiles of the Fe I 630.15 and $630.25 \mathrm{~nm}$ (top) and Ca II $854.2 \mathrm{~nm}$ lines (bottom), computed within the white box shown on the left panel. The observations were acquired at the line positions marked with circles.
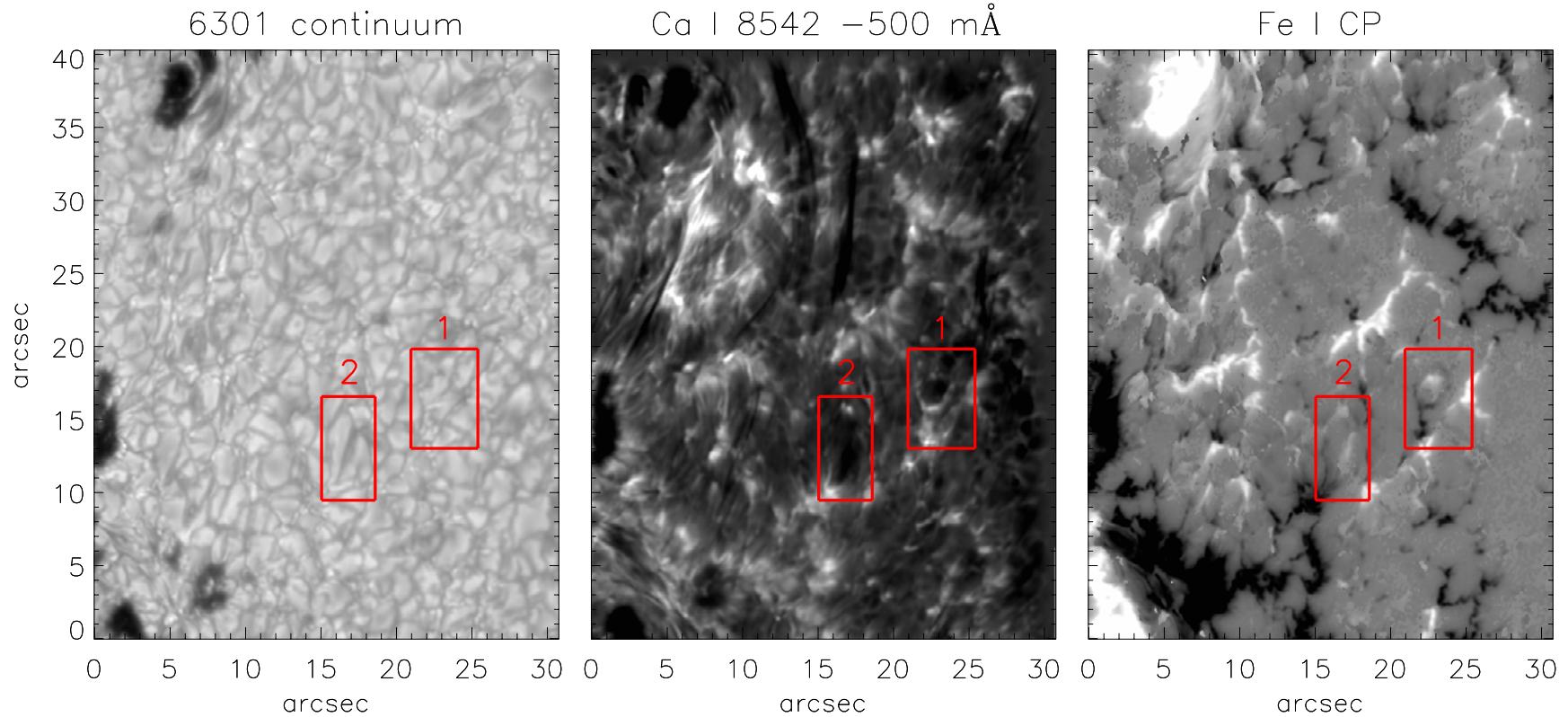

Figure 3. SST observations of NOAA 11024 on 2009 July 5 at 10:13:14 UT. The $30^{\prime \prime} \times 40^{\prime \prime}$ FOV shown here focuses on the area where magnetic flux was emerging more intensively. Left: continuum intensity at $630.32 \mathrm{~nm}$. Middle: Ca II $854.2 \mathrm{~nm}$ Stokes $I$ filtergram at $-0.05 \mathrm{~nm}$ from the line center. Right: mean circular polarization degree in the Fe I lines (scaled to $\pm 5 \%$ ). The red boxes outline the FOVs corresponding to each of the two emergence events analyzed in this paper (cases 1 and 2).

(A color version of this figure is available in the online journal.)

magnetic signals are smaller than $3 \sigma$, with $\sigma$ the noise of the photospheric magnetograms. Then we averaged the bisectors between the $40 \%$ and $70 \%$ intensity levels in the selected pixels and took the result as the zero point for the velocity scale.

A similar procedure was applied to the chromospheric line. In order to convert the Ca II $854.2 \mathrm{~nm}$ bisector positions to velocities, we again assumed that the quiet Sun pixels are at rest on average.

To study the evolution of the longitudinal and transverse components of the vector magnetic field, we use the mean linear polarization degree (LP),

$$
\mathrm{LP}=\frac{\int_{\lambda_{\mathrm{b}}}^{\lambda_{\mathrm{r}}}\left[Q^{2}(\lambda)+U^{2}(\lambda)\right]^{1 / 2} / I(\lambda) \mathrm{d} \lambda}{\int_{\lambda_{b}}^{\lambda_{r}} \mathrm{~d} \lambda},
$$

and the mean circular polarization degree $(\mathrm{CP})$,

$$
\mathrm{CP}=\frac{\int_{\lambda_{\mathrm{b}}}^{\lambda_{\mathrm{r}}}|V(\lambda)| / I(\lambda) \mathrm{d} \lambda}{\int_{\lambda_{b}}^{\lambda_{\mathrm{r}}} \mathrm{d} \lambda},
$$


where $\lambda_{\mathrm{r}}$ and $\lambda_{\mathrm{b}}$ represent the limits of integration over the line. The LP and CP have been calculated for the two Fe I lines separately and then averaged in order to improve the signal-to-noise ratio.

\subsection{Retrieval of the Magnetic Properties of the Bubble}

The four Stokes profiles of the Fe I lines were inverted simultaneously to determine the magnetic and dynamic properties of the fields emerging in the photosphere. To this end, we used the SIR inversion code (Stokes Inversion based on Response functions; Ruiz Cobo \& del Toro Iniesta 1992) and a simple one-component model atmosphere. The atmospheric parameters were taken to be constant with height except for the temperature, whose stratification was obtained by perturbing the Harvard Smithsonian Reference Atmosphere (Gingerich et al. 1971) at two specific optical depths called nodes. In view of the high spatial resolution attained by our observations, we set the magnetic filling factor to unity and did not use any macroturbulence or stray light contamination. In total, the inversion returned the values of seven free parameters (the three components of the vector magnetic field, the line of sight (LOS) velocity, the temperature at the two nodes, and the microturbulence) for each pixel within the observed FOV.

The longitudinal component of the chromospheric magnetic field was computed from the Ca II $854.2 \mathrm{~nm}$ Stokes $V$ profiles by using the weak field approximation. We can apply this approximation here because the Zeeman splitting is much smaller than the thermal width of the line. However, the magnetic field may change over the line formation region, so the method remains qualitative. In the weak field regime, $V(\lambda)$ is related to the longitudinal component of the field through the following relationship

$$
V(\lambda)=-\phi C \frac{\delta I}{\delta \lambda},
$$

(e.g., Landi degl'Innocenti \& Landolfi 2004), where $\phi=$ $f B \cos (\gamma)$ is the longitudinal flux density, $f$ is the magnetic filling factor, $B$ is the field strength, $\gamma$ is the field inclination with respect to the LOS, $C=4.67 \times 10^{-13} \lambda_{0}^{2} g$ is a constant that depends on the central wavelength $\lambda_{0}$ and the effective Landé factor $g$ of the transition, and $\delta I / \delta \lambda$ is the derivative of the intensity profile with wavelength; $\phi$ is in $\mathrm{Mx} \mathrm{cm} \mathrm{cm}^{-2}$ when $\lambda_{0}$ is given in $\AA$. We have followed the least-squares method used by Martínez González \& Bellot Rubio (2009) to obtain $\phi$ as

$$
\phi=-\frac{\Sigma_{i} \frac{\delta I}{\delta \lambda_{i}} V_{i}}{C \Sigma_{i}\left(\frac{\delta I}{\delta \lambda_{i}}\right)^{2}},
$$

where the subscript $i$ labels the $i$ th wavelength sample.

\section{RESULTS}

In this section, we describe two characteristic flux emergence events and the various phenomena that occur simultaneously as the magnetic field crosses the photosphere and intrudes at least into the mid chromosphere.

After a global description of the two emergence events based on the recorded filtergrams, we will perform a more detailed analysis of event 1 that will include a discussion of the properties of the observed Stokes profiles and a derivation of LOS velocities and magnetic fields both in the photosphere and in the chromosphere.

\subsection{The Dark Bubble and Other Signs of Flux Emergence}

Figure 4 displays a temporal sequence of CP maps (top panels) and Ca II 854.2-0.08 nm filtergrams (bottom panels) for flux emergence event 1 . The UT times indicated in each panel are those of the first wavelength point of the corresponding Fe I $630 \mathrm{~nm}$ or Ca II $854.2 \mathrm{~nm}$ line scan.

During the sequence, which spans about 10 minutes, a negative polarity patch showing a semi-circular shape is observed. In the third magnetogram of the sequence, a weak positive blob appears at $(x, y)=\left(2^{\prime \prime}, 4^{\prime \prime}\right)$. This magnetic knot rapidly grows from the third to the fourth magnetogram, reaching a size of $0.5 \times 0.5$. The subsequent evolution is dominated by the separation of the opposite magnetic polarities, as indicated by the arrows. At 10:09:42 UT, the centers of the two polarities are separated by 1".4. This distance grows steadily up to 3.6 at 10:17:48 UT. The separation speed reaches a maximum of $5.0 \mathrm{~km} \mathrm{~s}^{-1}$ at 10:14:20 UT and slows down to $2.7 \mathrm{~km} \mathrm{~s}^{-1}$ at the end of the sequence.

The bottom panels of Figure 4 show the corresponding $\mathrm{Ca}$ II intensity images. The arrows still refer to the magnetic legs described above. Brightenings in the intensity sequence resemble the shape of and coincide in position with the magnetic features seen in the magnetograms. Particularly bright areas are observed from 10:13:56 UT until the end of the sequence. However, the most striking feature in the intensity images is the occurrence of a dark bubble, which occupies a more or less circular area within the limits defined by the magnetic feet. For the purpose of detection, we have defined the dark bubble as those pixels - within the mentioned limits-whose intensity in the Ca II $854.2-0.08 \mathrm{~nm}$ filtergram is $\leqslant 0.7 I_{\mathrm{c}}$. When we use this criterion, the bubble turns out to be visible from 10:09:42 to 10:13:14 UT (magnetogram time), i.e., for four line scans. The dark bubble grows from $1^{\prime \prime} .5 \times 0^{\prime \prime} .6$ at 10:09:42 UT to $1^{\prime \prime} .7 \times 1^{\prime \prime} .2$ at 10:13:14 UT. The evolution of the bubble size (the $a_{1}$ and $a_{2}$ parameters) as well as the separation between the centroids of the opposite-polarity magnetic legs (the $b$ parameter) is summarized in Table 1 and Figure 5. We find a more or less steady increase in the $a_{2}$ direction but an intermittent increase in the $a_{1}$ direction. This is also reflected in the expansion velocity, which is steady along the $a_{2}$ axis (from 1.1 to $3 \mathrm{~km} \mathrm{~s}^{-1}$ ) but can take both positive and negative values along the $a_{1}$ axis. Note, for example, the negative expansion velocity of $-3.9 \mathrm{~km} \mathrm{~s}^{-1}$ at 10:12:01 UT.

The evolution of flux emergence event 2 is shown in Figure 6. Only the CaII $854.2 \mathrm{~nm}$ intensity filtergrams are displayed, but the details of the magnetic feet separation and the bubble growth can be found in Table 2 and Figure 5. The sequence lasts for 12 minutes, and the general behavior is very similar to case 1 . The arrows pinpoint the magnetic feet of opposite polarity as they separate from each other during the emergence process. In this case, the feet reach a maximum separation of 6.' 6 after 10 minutes. The dark bubble grows in size but in a rather discontinuous way, suggesting that the emergence of flux occurs in the form of successive jumps with intermediate stops. The two events considered here show the same behavior. The horizontal expansion velocity of the dark bubble peaks at $7 \mathrm{~km} \mathrm{~s}^{-1}$, with an average of $1 \mathrm{~km} \mathrm{~s}^{-1}$.

To get a better impression of how these events evolve with height, Figure 7 shows a temporal sequence of observables formed at various heights for flux emergence case 1 (see also the accompanying movie in the electronic edition of the Journal). The sequence spans 10 minutes. From left to right, the following parameters are displayed: continuum intensity at 

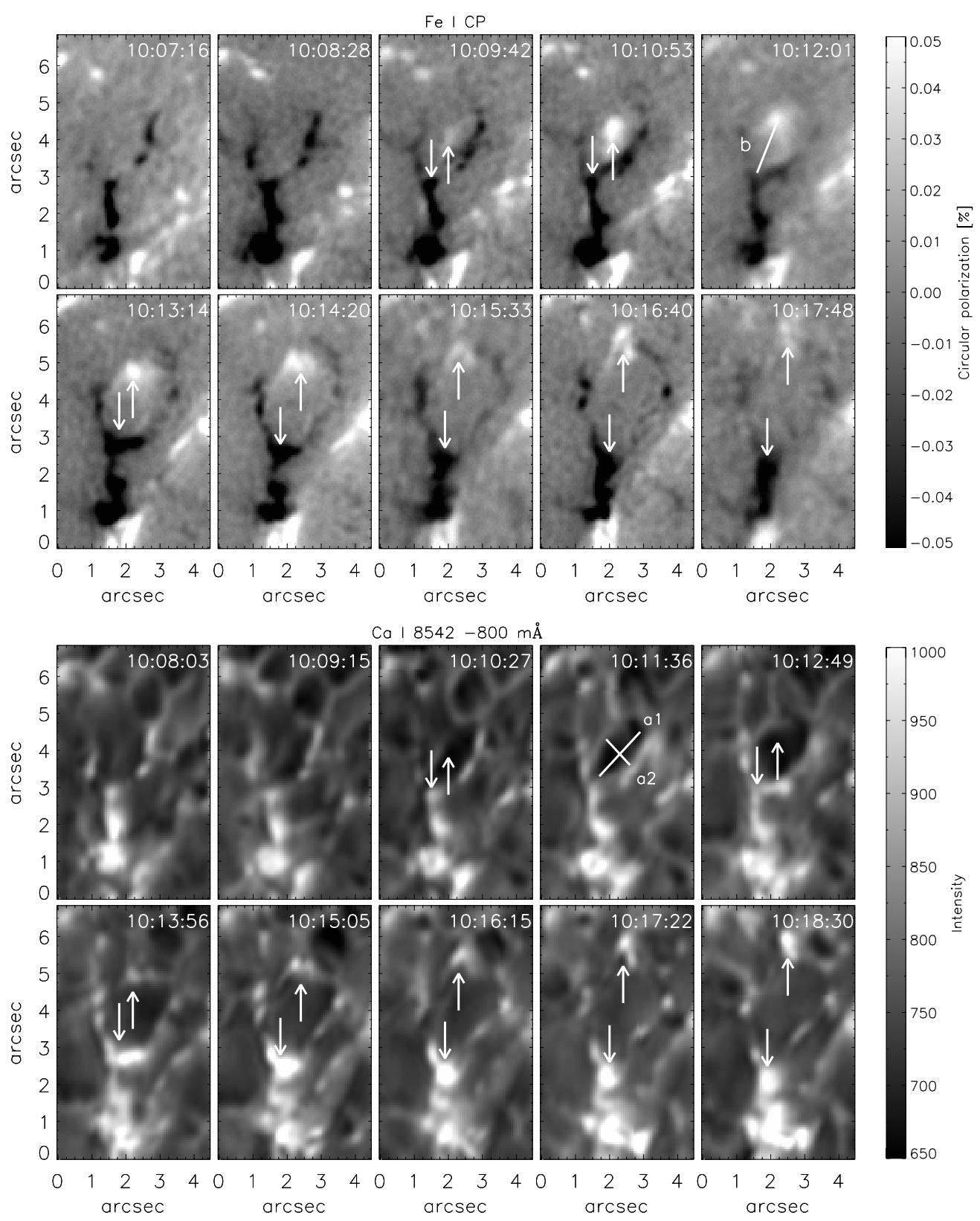

Figure 4. Top: temporal evolution of the photospheric magnetograms corresponding to the first case of flux emergence. Times in UT are indicated in the upper right corner of each panel. The sequence shows the appearance of a positive polarity foot (white) close to an already existing negative polarity semi-circular patch (black). The arrows indicate feet of opposite polarity as they separate from each other during the emergence process. Bottom: same as above for the Ca II $854.2-0.08 \mathrm{~nm}$ intensity filtergrams. Here the arrows still highlight the magnetic legs. Major intensity enhancements are associated with the negative leg. Some of the brightenings [located around $\left.(x, y)=\left(2^{\prime \prime}, 0^{\prime \prime} 5\right)\right]$ appear when the negative foot interacts with a preexisting positive patch. The dark bubble is well visible in the intensity images from 10:10:27 to 10:13:56 UT; $a_{1}$ and $a_{2}$ are its dimensions, and $b$ is the distance between the magnetic legs of opposite polarity (Tables 1 and 2).

$630.32 \mathrm{~nm}$, photospheric LP and CP maps, and seven filtergrams across the CaII $854.2 \mathrm{~nm}$ line from -0.06 to $+0.06 \mathrm{~nm}$ in steps of $0.02 \mathrm{~nm}$. Figure 1 provides an approximate translation of these line positions into heights. The green contours in the LP and CP panels indicate a mean linear polarization degree of $0.6 \%$. The red contours shown on the $\mathrm{CP}$ map represent mean circular polarization degrees of $1.3 \%$. The yellow contours highlight the presence of the dark bubble during its lifetime. They are plotted only in columns 1 and 4 to avoid cluttering.

Figure 7 illustrates the various phenomena occurring as the magnetic flux rises through the solar atmosphere. The most remarkable is the formation of a dark bubble. The continuum intensity at $630.2 \mathrm{~nm}$ does not show any traces of the dark bubble, as can be seen in the first column. However, other effects of the emergence are evident in the photosphere, such as abnormal granulation. At the position of the emerging flux the granules become bigger and more elongated than usual, e.g., near $(x, y)=\left(1^{\prime \prime} .5,4^{\prime \prime} .5\right)$ at 10:07:16 UT.

The dark bubble rises through the solar atmosphere. This is clearly inferred from our sampling of the Ca II $854.2 \mathrm{~nm}$ line because we observe the bubble to gradually move to the line center as time progresses. The dark bubble first appears at 10:09:42 UT in the Ca II 854.2-0.06 and -0.04 nm filtergrams, that is, the mid photospheric wings of the line. However, in the next scan at 10:10:53 UT, it is also detected in the Ca II 854.2 $-0.02 \mathrm{~nm}$ filtergram and has increased in size. Subsequently, the dark bubble grows (see Table 1) and shows up closer and 

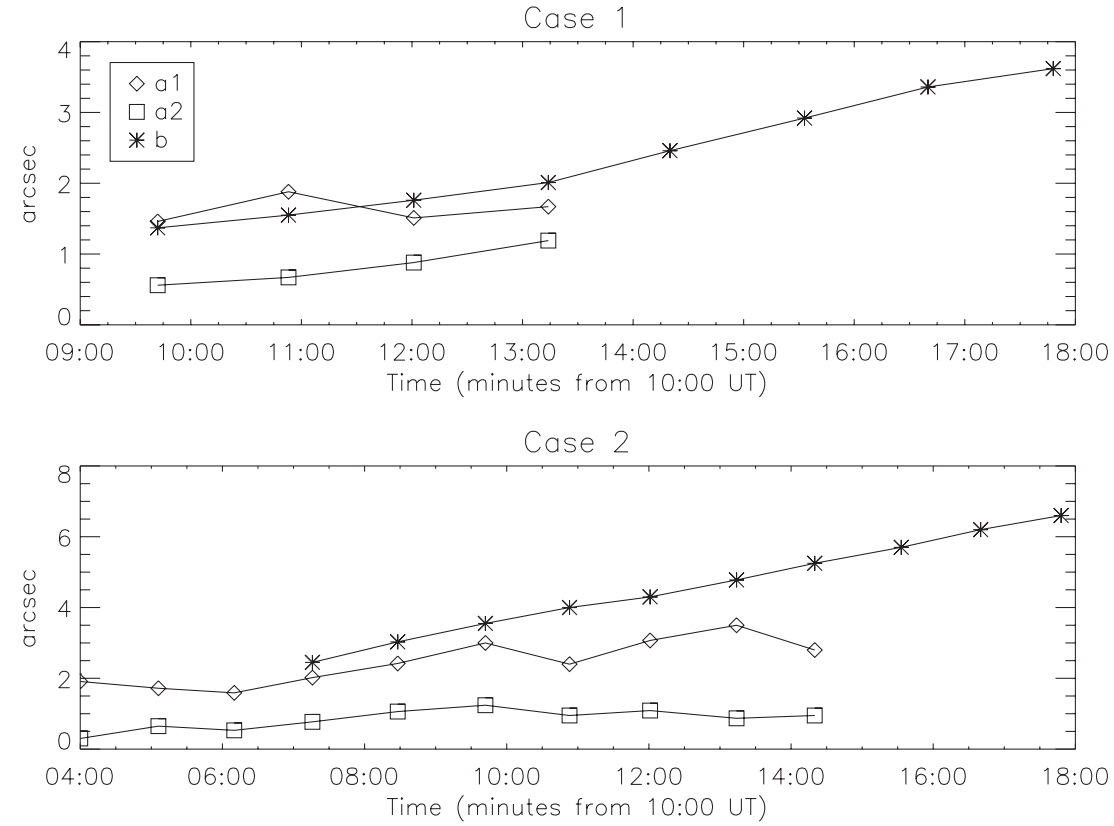

Figure 5. Temporal evolution of the $a_{1}, a_{2}$, and $b$ parameters for cases 1 and 2. Parameters $a_{1}$ and $a_{2}$ are the dimensions of the dark intensity bubble as depicted in Figure 4. The bubble is defined as the pixels with Ca II intensities equal to $0.7 I_{\mathrm{c}}$ or less that are within the area enclosed by the magnetic feet. Parameter $b$ is the distance between magnetic legs of opposite polarity, also depicted in Figure 4 (see Tables 1 and 2 for numerical values).

Table 1

Properties of Ca II 854.2 Bubble 1

\begin{tabular}{|c|c|c|c|c|c|c|c|}
\hline \multirow{2}{*}{$\begin{array}{l}\text { Time } \\
\text { (UT) }\end{array}$} & \multirow{2}{*}{$\begin{array}{l}\text { Bubble Size } \\
\left(a_{1} \times a_{2}\right)\end{array}$} & \multirow{2}{*}{$\begin{array}{c}\text { Magnetic Feet Separation } \\
\qquad(b)\end{array}$} & \multicolumn{2}{|c|}{ Bubble Horizontal Expansion } & \multirow{2}{*}{$\begin{array}{l}\text { Magnetic Feet Horizontal Speed } \\
\qquad\left(\mathrm{km} \mathrm{s}^{-1}\right)\end{array}$} & \multirow{2}{*}{$\begin{array}{l}\text { Vertical Speed } \\
\qquad\left(m \AA \mathrm{s}^{-1}\right)\end{array}$} & \multirow{2}{*}{$\begin{array}{l}\text { Rising Time } \\
\text { (s) }\end{array}$} \\
\hline & & & $\left(a_{1}, \mathrm{~km} \mathrm{~s}^{-1}\right)$ & $\left(a_{2}, \mathrm{~km} \mathrm{~s}^{-1}\right)$ & & & \\
\hline 10:07:16 & $\cdots$ & $\ldots$ & $\cdots$ & $\cdots$ & $\cdots$ & $\cdots$ & $\cdots$ \\
\hline $10: 08: 28$ & $\ldots$ & $\ldots$ & $\ldots$ & $\ldots$ & $\ldots$ & $\ldots$ & $\ldots$ \\
\hline 10:09:42 & $1^{\prime \prime} 46 \times 00^{\prime \prime} 56$ & $1^{\prime \prime} 37$ & $\ldots$ & $\ldots$ & $\ldots$ & $\ldots$ & $\ldots$ \\
\hline $10: 10: 53$ & $1^{\prime \prime} .88 \times 00^{\prime \prime} 67$ & $1^{\prime \prime} 55$ & 4.23 & 1.10 & 1.81 & 1.38 & 72 \\
\hline 10:12:01 & $1^{\prime \prime} .51 \times 00^{\prime \prime} 88$ & 1".76 & -3.88 & 2.20 & 2.20 & 1.45 & 69 \\
\hline $10: 13: 14$ & $1^{\prime \prime} 67 \times 1^{\prime \prime} .19$ & $2^{\prime \prime} 01$ & 1.56 & 3.03 & 2.45 & 1.35 & 74 \\
\hline 10:14:20 & $\ldots$ & 2.46 & $\ldots$ & $\ldots$ & 4.86 & $\ldots$ & $\ldots$ \\
\hline $10: 15: 33$ & $\ldots$ & 2.92 & $\ldots$ & $\ldots$ & 4.50 & $\ldots$ & $\ldots$ \\
\hline $10: 16: 40$ & $\ldots$ & 3."36 & $\ldots$ & $\ldots$ & 4.69 & $\ldots$ & $\ldots$ \\
\hline $10: 17: 48$ & $\ldots$ & 3." 62 & $\ldots$ & $\ldots$ & 2.73 & $\ldots$ & $\ldots$ \\
\hline
\end{tabular}

Notes. Column 1: time of the first wavelength of a full scan. Column 2: size of dark bubble as measured in Ca II intensity. The bubble is defined by the intensities of less than $0.7 I_{\mathrm{c}}$ that are within the area enclosed by the magnetic feet. Dimensions $a_{1}$ and $a_{2}$ are defined in Figure 4 and displayed in Figure 5. Column 3: distance between the opposite-polarity magnetic feet. The separation $b$ is defined in Figure 4 and summarized in Figure 5. Column 4: horizontal expansion of the bubble area in the $a_{1}$ and $a_{2}$ directions relative to the previous scan. Column 5: speed of separation of the opposite-polarity magnetic feet. Column 6: vertical speed of the bubble measured from one wavelength position (or height) to the next. Column 7: bubble rising time from one wavelength position (or height) to the next. Missing information is marked with ellipses.

closer to the line core. The pattern is similar in the red wing of the line.

The third column of Figure 7 shows patches of enhanced circular polarization (red contours) from the beginning of the sequence in spatial coincidence with the magnetic feet of negative polarity. At 10:09:42 UT, a small patch of linear polarization appears right next to where the positive polarity patch is emerging (see top panels of Figure 4 for a more detailed view). The LP patch has grown at 10:10:53 UT and is now in between the opposite polarities of the bipole. This implies the rising of a horizontal magnetic field in photospheric layers, with more vertical legs anchored deeper down. At 10:12:01 UT, the LP patch has decreased in size but still remains between the opposite-polarity feet. At 10:13:14 UT, the linear polarization signal is almost washed out, whereas the vertical field is visible until the end of the sequence. The dark bubble mimics the behavior of the LP patch: it emerges, increases in size, and lasts for the same duration, disappearing at 10:13:14 UT. The dark bubble criterion is only satisfied for four time scans, from 10:09:42 UT until 10:13:14 UT. Beyond 10:13:14 UT, we can still see ring-shaped brightenings that coincide with the magnetic legs. Those are the boundaries of the magnetic bubble. Overall, the observations indicate that the dark bubble is a counterpart, in higher layers, of the appearance of horizontal fields in the photosphere.

The evolution of the magnetic bubble is dominated by the separation of the opposite magnetic polarities (column 3 of Figure 7). The magnetic bubble seems to leave footprints in the chromospheric intensity images, and brightenings are seen at all wavelengths of the Ca II $854.2 \mathrm{~nm}$ scan, resembling the shape and position of the magnetic features observed in the photospheric magnetograms. 


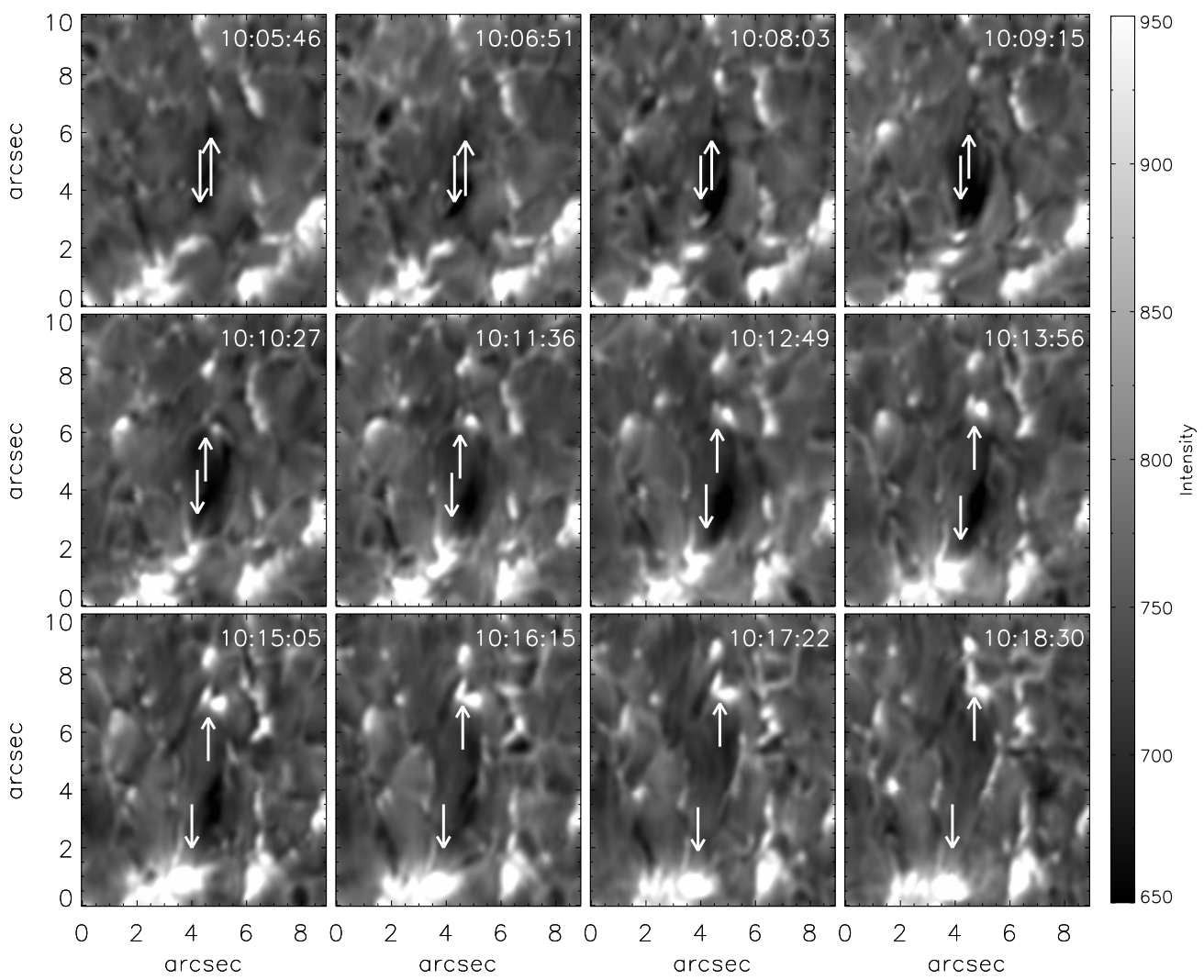

Figure 6. Same as Figure 4 for the second example of flux emergence. In this case, only the Ca II $854.2 \mathrm{~nm}$ intensity filtergrams are shown. The arrows pinpoint the opposite-polarity magnetic feet as they separate from each other during the emergence process. Note the brightenings at $(x, y)=\left(1^{\prime \prime}-4^{\prime \prime}, 0^{\prime \prime}-2^{\prime \prime}\right)$. In this event, the dark bubble is more elongated and lasts during the whole 12 minute sequence.

Table 2

Properties of Ca II 854.2 Bubble 2

\begin{tabular}{|c|c|c|c|c|c|c|c|}
\hline \multirow{2}{*}{$\begin{array}{l}\text { Time } \\
\text { (UT) }\end{array}$} & \multirow{2}{*}{$\begin{array}{c}\text { Bubble Size } \\
\left(a_{1} \times a_{2}\right)\end{array}$} & \multirow{2}{*}{$\begin{array}{l}\text { Magnetic Feet Separation } \\
\qquad(b)\end{array}$} & \multicolumn{2}{|c|}{ Bubble Horizontal Expansion } & \multirow{2}{*}{$\begin{array}{l}\text { Magnetic Feet Horizontal Speed } \\
\qquad\left(\mathrm{km} \mathrm{s}^{-1}\right)\end{array}$} & \multirow{2}{*}{$\begin{array}{l}\text { Vertical Speed } \\
\qquad\left(\mathrm{m} \AA \mathrm{s}^{-1}\right)\end{array}$} & \multirow{2}{*}{$\begin{array}{c}\text { Rising Time } \\
\text { (s) }\end{array}$} \\
\hline & & & $\left(a_{1}, \mathrm{~km} \mathrm{~s}^{-1}\right)$ & $\left(a_{2}, \mathrm{~km} \mathrm{~s}^{-1}\right)$ & & & \\
\hline 10:04:00 & $1^{\prime \prime} .91 \times 0.130$ & $\ldots$ & $\ldots$ & $\ldots$ & $\ldots$ & $\ldots$ & $\ldots$ \\
\hline 10:05:06 & $1^{\prime \prime} 72 \times 00^{\prime \prime} 65$ & $\ldots$ & -2.00 & 3.78 & $\ldots$ & $\ldots$ & $\ldots$ \\
\hline 10:06:10 & $1^{\prime \prime} .59 \times 00^{\prime \prime} .53$ & $\ldots$ & -1.45 & -1.33 & $\ldots$ & 1.53 & 65 \\
\hline 10:07:16 & $2^{\prime \prime} .02 \times 00^{\prime \prime} .77$ & 2.45 & 4.65 & 2.59 & $\ldots$ & 1.50 & 67 \\
\hline 10:08:28 & $2^{\prime \prime} .42 \times 1^{\prime \prime} .06$ & 3."03 & 3.97 & 2.88 & 5.76 & $\ldots$ & $\ldots$ \\
\hline 10:09:42 & $3{ }^{\prime \prime} 00 \times 11^{\prime \prime} .24$ & 3.'55 & 5.60 & 1.74 & 5.02 & $\cdots$ & $\cdots$ \\
\hline 10:10:53 & $2^{\prime \prime} .40 \times 0^{\prime \prime} .95$ & 4. & -6.00 & -3.00 & 4.53 & $\ldots$ & $\ldots$ \\
\hline 10:12:01 & $3 . .07 \times 11^{\prime \prime} 09$ & 4.30 & 7.00 & 1.50 & 3.15 & $\ldots$ & $\ldots$ \\
\hline 10:13:14 & $3^{\prime \prime} 50 \times 0^{\prime \prime} 87$ & 4.78 & 4.21 & -2.15 & 4.70 & $\ldots$ & $\ldots$ \\
\hline 10:14:20 & $2^{\prime \prime} .80 \times 00^{\prime \prime} .95$ & 5.25 & -7.57 & 0.86 & 5.08 & $\ldots$ & $\ldots$ \\
\hline 10:15:33 & $\ldots$ & 5.70 & $\ldots$ & $\ldots$ & 4.40 & $\ldots$ & $\ldots$ \\
\hline 10:16:40 & $\ldots$ & 6.20 & $\ldots$ & $\ldots$ & 5.30 & $\ldots$ & $\ldots$ \\
\hline 10:17:48 & $\ldots$ & $6 . " 60$ & $\ldots$ & $\ldots$ & 4.20 & $\ldots$ & $\ldots$ \\
\hline
\end{tabular}

Notes. Same as Table 1 for flux emergence event 2.

To show how the dark bubble makes its way through the solar atmosphere in more detail, Figure 8 presents a close-up of the blue wing of the Ca II $854.2 \mathrm{~nm}$ line. We only consider the time interval during which the dark bubble exists, i.e., from 10:09:42 UT to 10:13:14 UT, plus two time steps before its appearance for completeness. The first column shows maps of the photospheric circular polarization to illustrate the presence and evolution of the magnetic bubble. The next columns display, respectively, Ca II $854.2 \mathrm{~nm}$ filtergrams at $-0.06,-0.04,-0.03$, $-0.02,-0.01$, and $0 \mathrm{~nm}$ from the line center. Footprints of the magnetic bubble can be clearly seen at all wavelengths in Ca II
$854.2 \mathrm{~nm}$ in the form of brightenings. White arrows point to the dark bubble when it is unambiguously detected, while yellow arrows indicate a more vague presence of the bubble. In the latter cases, we do not directly observe the dark bubble, but we infer its presence from the circle-shaped brightenings that coincide with the magnetic legs. An example can be found in the line core filtergram at 10:13:14 UT. As the line core is approached, opaque chromospheric features (fibrils), presumably located at greater heights, appear in the FOV and do not allow one to see deep in the atmosphere. In spite of this, we detect the dark bubble at 10:09:42 UT in the Ca II 854.2 -0.06 nm filtergram and, one 


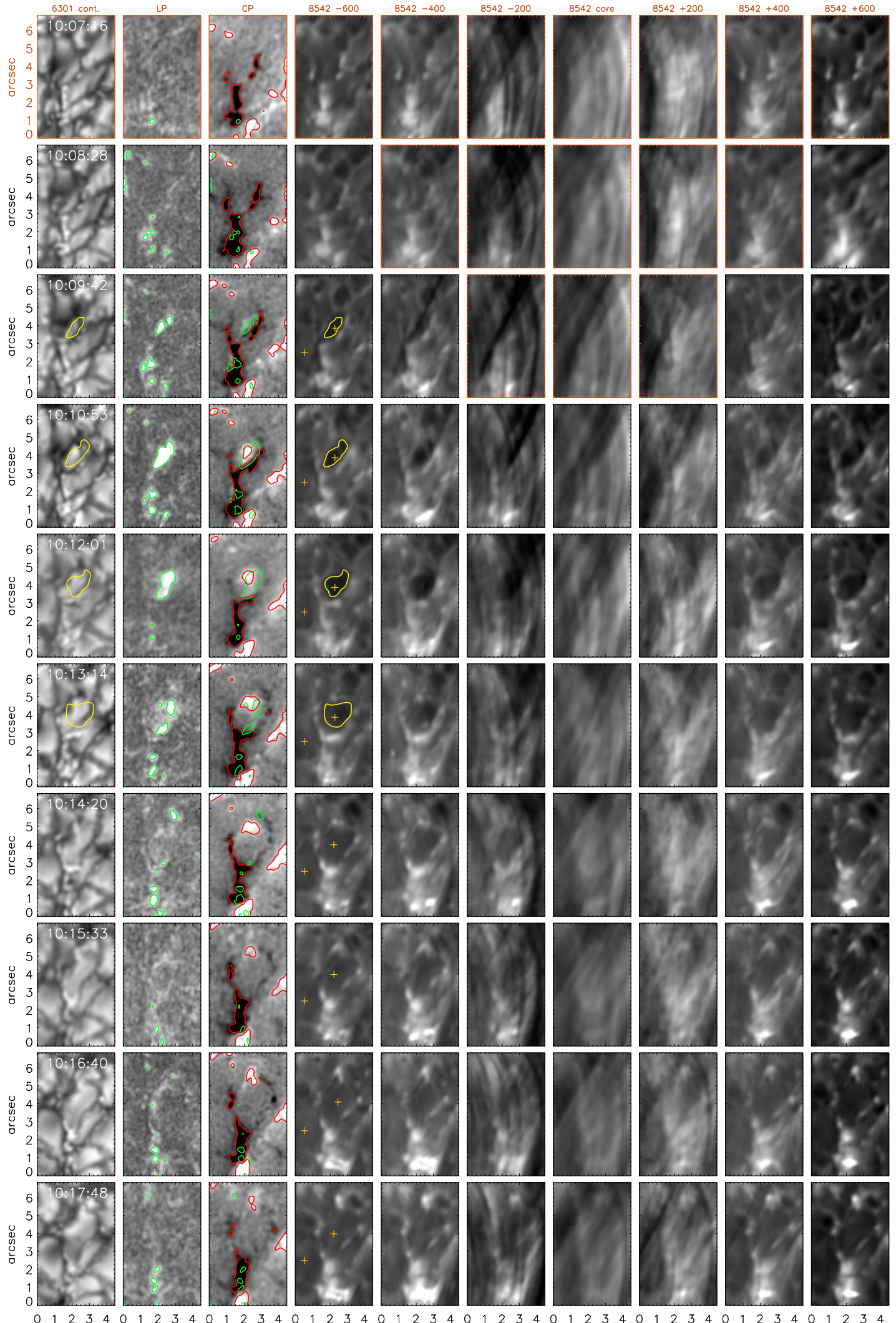

Figure 7. Temporal evolution of several observables corresponding to flux emergence case 1. From left to right: continuum intensity at $630 \mathrm{~nm}$, photospheric LP and $\mathrm{CP}$ maps, and filtergrams across the Ca II $854.2 \mathrm{~nm}$ line from -0.06 to $+0.06 \mathrm{~nm}$ in steps of $0.02 \mathrm{~nm}$. The green contours overplotted on the LP and CP panels indicate linear polarization signals of $0.6 \%$ of the $I_{\mathrm{c}}$. The red contours shown on the CP maps represent circular polarization signals of $1.3 \%$. The yellow contours mark the presence of the dark bubble during its life. Note that the dark bubble appears only in four line scans, while the magnetic bubble lives longer. Contours have been plotted only in two columns ( 1 and 4) to avoid overcrowding. Pixels marked with a yellow cross are those used in Figures 9 and 10 . The rise of the bubble through the atmosphere can be followed as it progresses from the wings to the core of Ca II $854.2 \mathrm{~nm}$. Panels with an orange frame are those in which the dark bubble is not yet visible.

(An animation and a color version of this figure are available in the online journal.) 

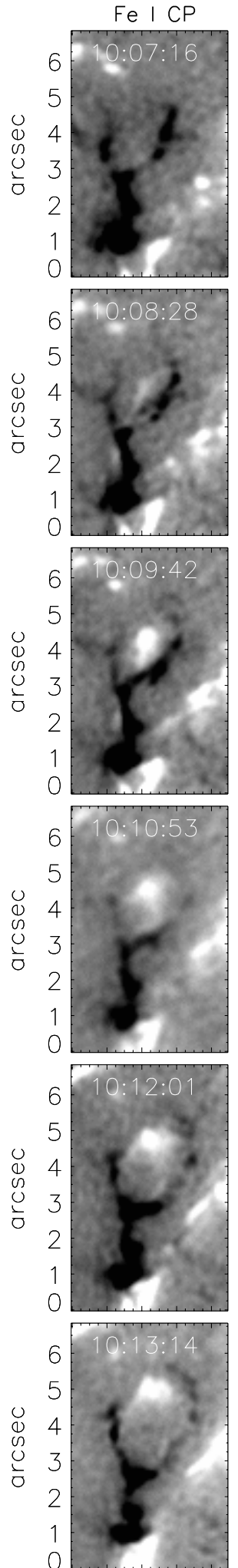

$0 \quad 12334$
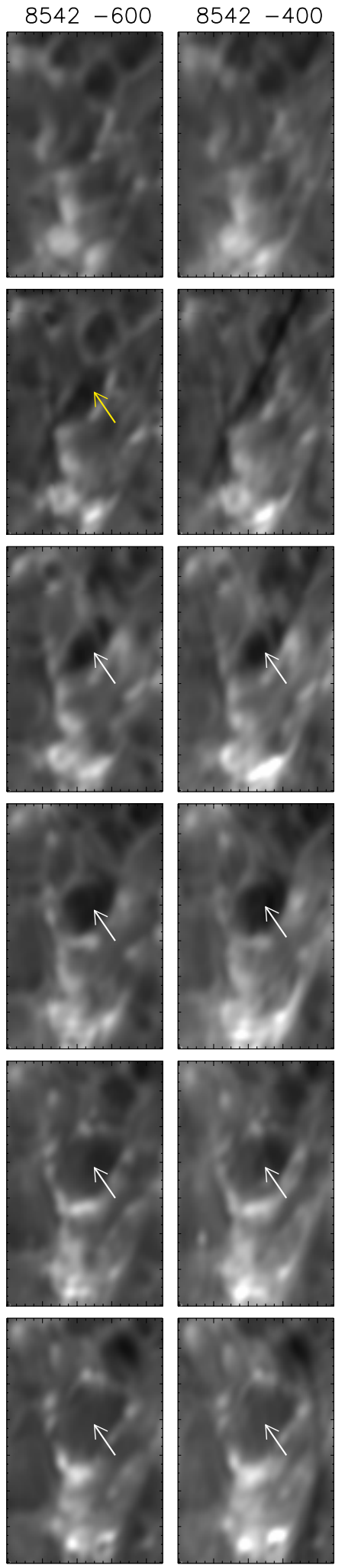
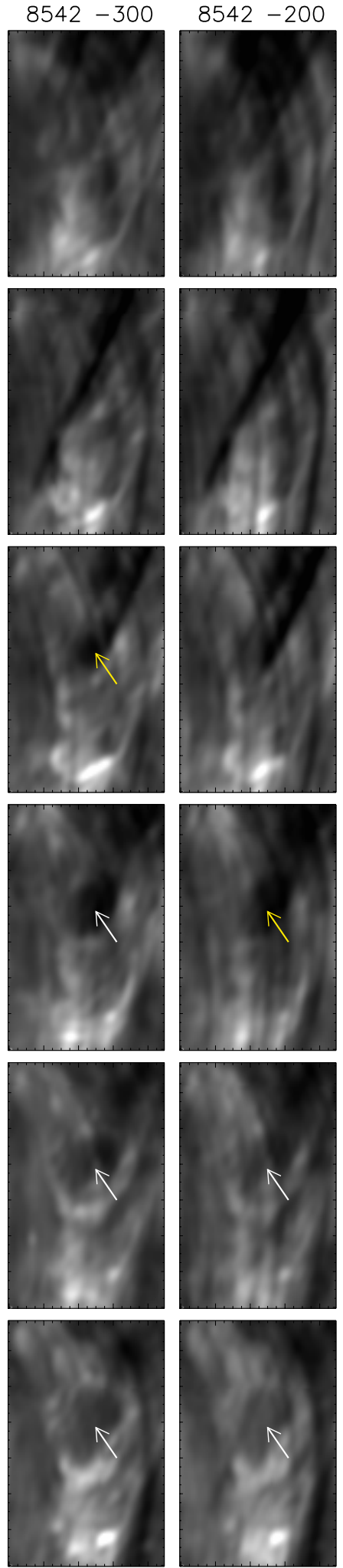

$8542-100$
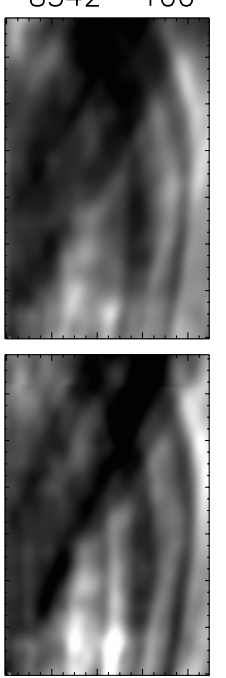

8542 core
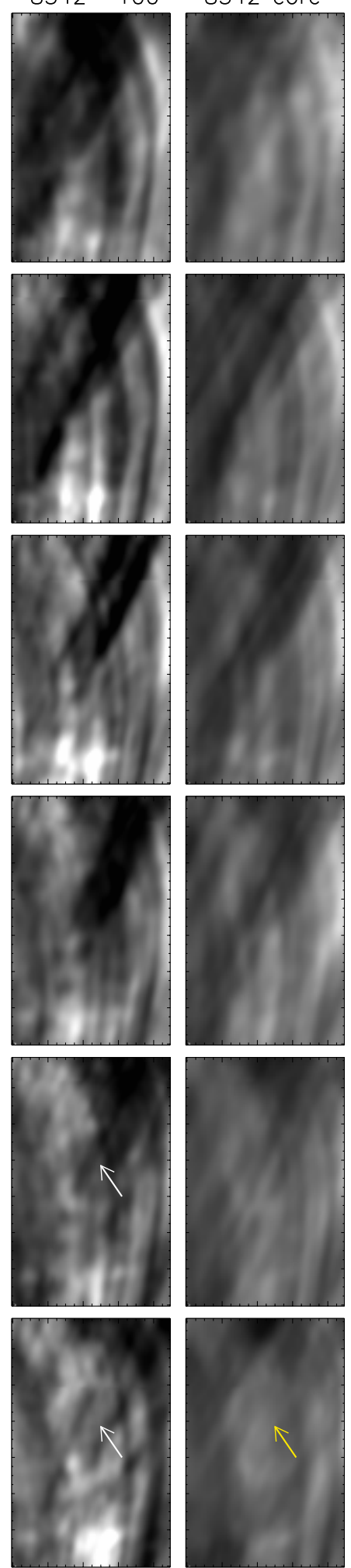

Figure 8. Close-up of the blue wing of the Ca II $854.2 \mathrm{~nm}$ line, from 10:07:16 UT to 10:13:14 UT. The first column shows photospheric circular polarization maps. The next columns display Ca II $854.2 \mathrm{~nm}$ filtergrams at $-0.06,-0.04,-0.03,-0.02,-0.01$, and $0.0 \mathrm{~nm}$ from the line core. White arrows indicate a clear detection of dark bubble 1. Yellow arrows mean a more ambiguous detection, either because the bubble is overlaid by chromospheric fibrils or because it is not a conspicuous dark feature. In the former case, the presence of the bubble can be inferred from the brightenings surrounding it.

(A color version of this figure is available in the online journal.) 

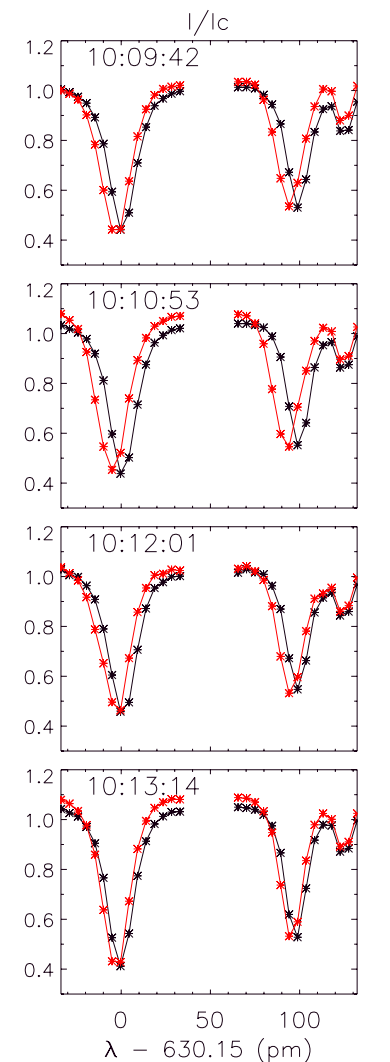
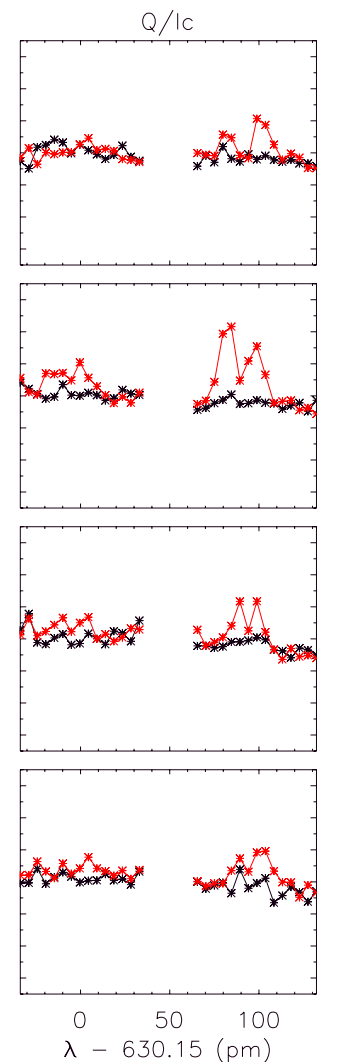
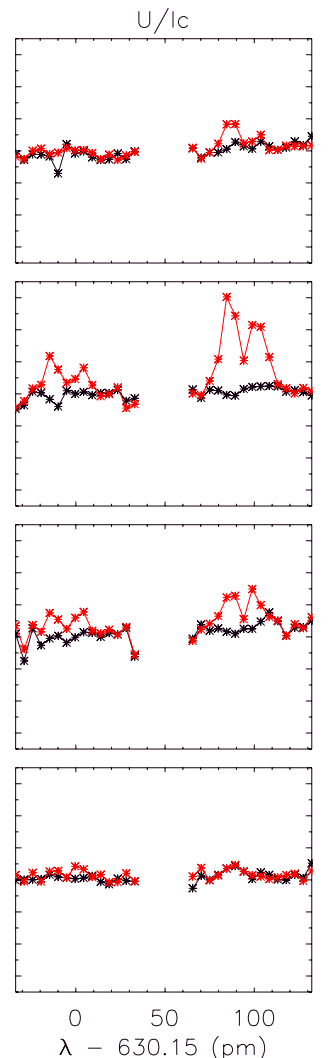
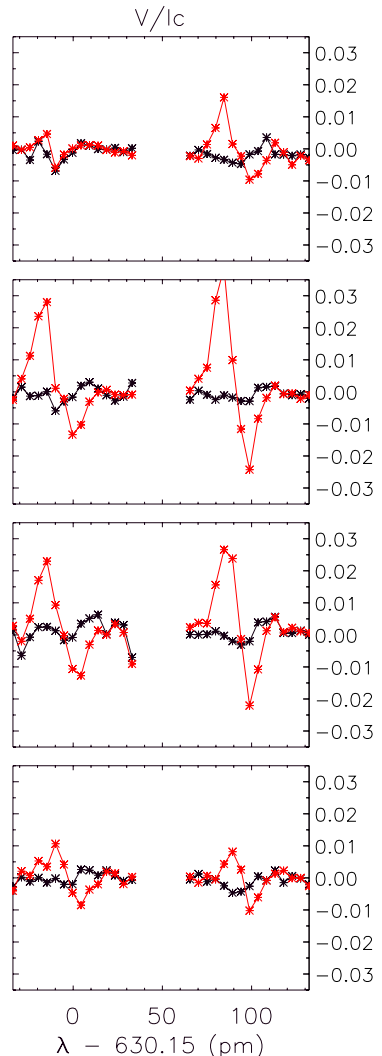

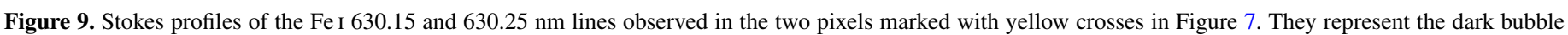

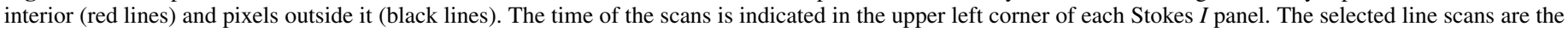
ones in which the dark bubble is more clearly visible (bottom panels of Figure 4) and in which significant LP signal is detectable (Figure 7).

(A color version of this figure is available in the online journal.)

minute later, also in Ca II 854.2-0.04 nm. At 10:10:53 UT, the bubble is still visible in those images, but it has progressed toward the Ca II $854.2-0.03 \mathrm{~nm}$ filtergram. At that time, by chance, the chromospheric fibrils seem to be on top of the dark bubble. By 10:12:01 UT, the bubble has reached the Ca II $854.2-0.01 \mathrm{~nm}$ filtergram, and at 10:13:14 UT it is detected at all wavelengths, including the line core. The behavior of the bubble in the red wing (not shown here but in Figure 7) is similar. The fact that the dark bubble approaches the core of the Ca II $854.2 \mathrm{~nm}$ line from the filtergram at $-0.06 \mathrm{~nm}$ means that it is traveling from the mid photosphere to the mid chromosphere over a distance estimated to be about $1100 \mathrm{~km}$ (Figure 1). Since it takes some $215 \mathrm{~s}$ for the dark bubble to get there, its average vertical speed is $5.2 \mathrm{~km} \mathrm{~s}^{-1}$.

\subsection{Polarization Profiles}

In this section, we discuss the properties of the observed Stokes profiles before extracting more quantitative information from them.

\subsubsection{Fe I $630 \mathrm{~nm}$ Lines}

Figure 9 shows the Fe I 630.15 and $630.25 \mathrm{~nm}$ Stokes profiles emerging from two different pixels, one inside and the other outside of dark bubble 1 (red and black lines, respectively). Those pixels have been marked with yellow crosses in Figure 7. Four time steps are displayed, corresponding to the initial phases of the dark bubble development (10:09:42, 10:10:53, 10:12:01, and 10:13:14 UT). As can be seen, all intensity profiles are blueshifted with respect to the pixel located outside of the magnetic bubble region. The blueshift grows from 10:09:42 UT, attains a maximum at 10:10:53 UT, and decreases in the following scans. This pattern confirms that the magnetic bubble is indeed rising in the atmosphere.

The Stokes $V$ profiles inside the emerging flux region show significant signals of up to $3.5 \% I_{\mathrm{c}}$, meaning that during the four line scans there was a magnetic field present in the bubble. They also exhibit very strong blueshifts. Stokes $Q$ and $U$, by contrast, have large amplitudes only at 10:10:53 and 10:12:01 UT. This agrees with Figure 7, where those two line scans are the ones showing the stronger and more extended LP patches. A clear Stokes $Q$ signal is detectable in the Fe I $630.25 \mathrm{~nm}$ line from 10:09:42 until 10:13:14 UT, which is consistent with the idea that at those particular times a horizontal magnetic field was entering and then leaving the line formation region in the photosphere.

The inversion of the Stokes profiles of the pixel inside the dark bubble yields field strengths of $480,610,360$, and $230 \mathrm{G}$ for each of the time steps depicted in Figure 9. The corresponding inclinations reveal a horizontal field becoming slightly more vertical: $87^{\circ}, 75^{\circ}, 72^{\circ}$, and $70^{\circ}$, respectively. Some single-lobed Stokes $V$ profiles are observed inside the dark bubble. Jumps or strong gradients of the physical parameters along the line of sight can explain such kind of profiles (Sainz Dalda et al. 2012), suggesting that a magnetic discontinuity is present in the atmosphere above the bubble.

\subsubsection{Ca II $854.2 \mathrm{~nm}$}

Figure 10 shows the temporal evolution of the Ca II $854.2 \mathrm{~nm}$ Stokes $I$ and $V$ spectra observed in the two pixels used above. 

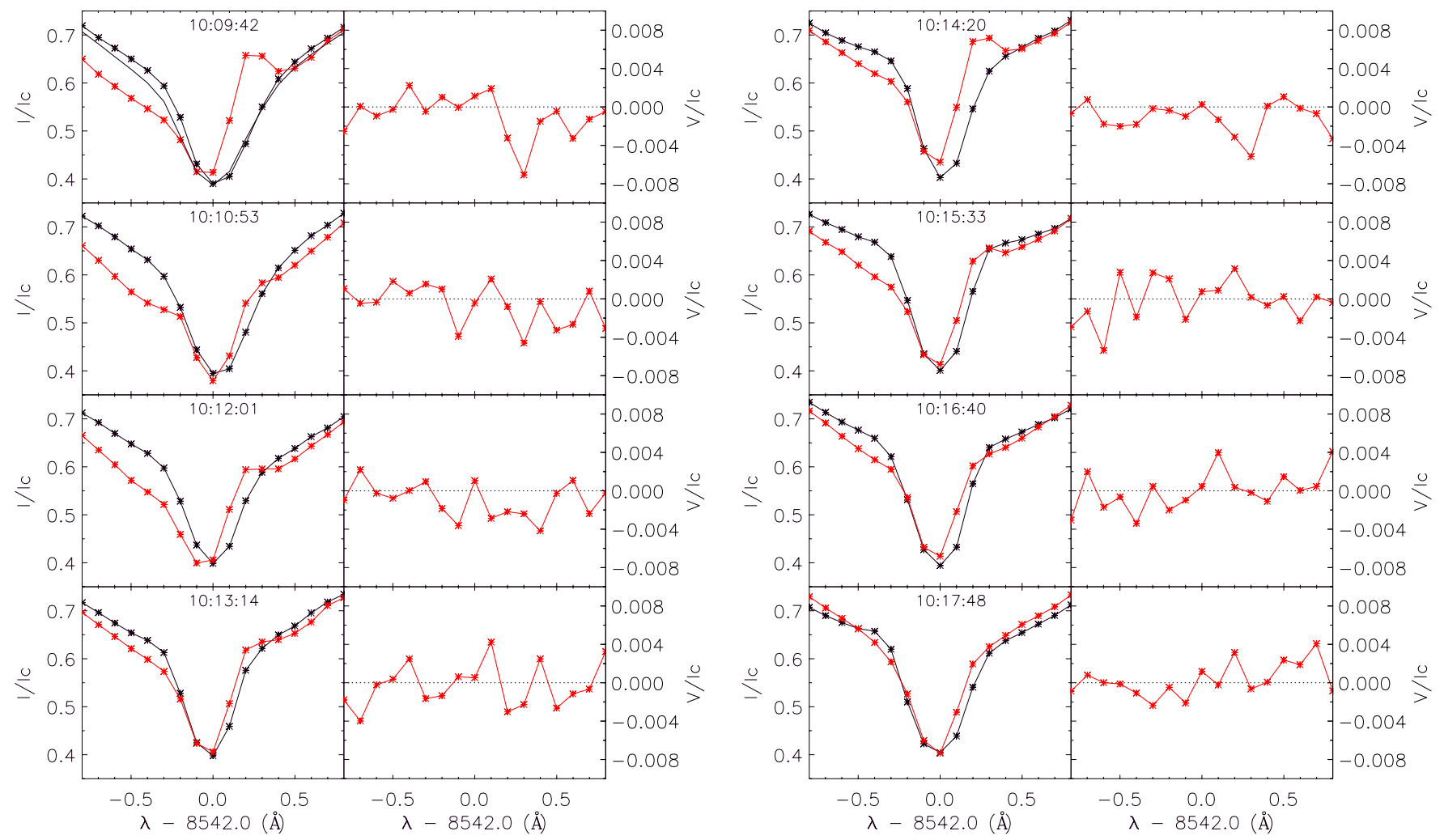

Figure 10. Temporal evolution of the Ca II 854.2 Stokes $I$ and $V$ profiles observed in two pixels, one inside dark bubble 1 (red lines) and another outside it (black lines). Those pixels have been highlighted with yellow crosses in Figure 7. A quiet Sun profile has been plotted for comparison in the first panel (simple black line). Note the clear blueshifts of $-2.9 \mathrm{~km} \mathrm{~s}^{-1}$ at 10:09:42 UT and $-2.7 \mathrm{~km} \mathrm{~s}^{-1}$ at 10:12:01 UT.

(A color version of this figure is available in the online journal.)

An average quiet Sun profile is overplotted for comparison (simple black line in the first panel). Note that regardless of time, the intensity profiles coming from the pixel outside the dark bubble and the quiet Sun are very similar.

Inside the dark bubble, the CaII $854.2 \mathrm{~nm}$ line shows enhanced absorption in the blue wing. Some of the profiles also show an emission peak (very prominent in particular at 10:09:42 and 10:14:20 UT). The peak lies at the "knee" of the line-roughly indicating an upper photospheric/low chromospheric origin - and occurs only in the red wing, not in both wings. This asymmetry implies the existence of very strong, localized upflows in layers below the formation height of the line core, which move the opacity blueward and expose deeper atmospheric layers but do not significantly shift the core itself. The mechanism is similar to that described by Leenaarts et al. (2010) in the first quartet of their Figure 9 or by Leenaarts et al. (2009) in their Figure 5, although the flows are of opposite direction here and probably less intense. To explain the emission feature, one may also need a strong temperature enhancement, but this will be investigated in Paper II of this series by using non-LTE inversions.

The intensity profiles observed in the bubble are strongly blueshifted at some particular times (10:09:42, 10:12:01, and 10:14:20 UT). Part of the blueshift might be induced by the presence of the emission peak, but part of it must be real because of the enhanced absorption occurring in the blue wing of the line.

Outside of the magnetic feet, the dark bubble exhibits no Ca II $854.2 \mathrm{~nm}$ Stokes $V$ signal above the noise level except at the wavelength of the intensity bump produced by the emission peak. Only there a weak chromospheric circular polarization signal can be measured. Although the profiles are very noisy, the existence of this signal is undisputable because it produces extended patches in the corresponding monochromatic Stokes $V$ images. We will see in Figure 13 that pixels with emission features are found mainly at the edges of the magnetic bubble, with only a few lying inside.

\subsection{Velocity of the Rising Gas}

In this section, we discuss the dynamics of the emerging bubble based on the bisector velocities derived from the observed Stokes $I$ profiles.

Figure 11 shows LOS velocity maps for flux emergence case 1 from 10:07:16 until 10:17:48 UT. The first and second rows display continuum intensity maps at $630.32 \mathrm{~nm}$ and Ca II 854.2 $-0.08 \mathrm{~nm}$ filtergrams, the third and fourth rows photospheric velocities at the $80 \%$ and $10 \%$ intensity levels, and the fifth and sixth rows chromospheric velocities at the $60 \%$ and $30 \%$ levels. The velocities are clipped at $\pm 2 \mathrm{~km} \mathrm{~s}^{-1}$ for the photosphere and at $\pm 4 \mathrm{~km} \mathrm{~s}^{-1}$ for the chromosphere. Negative velocities indicate blueshifts. Black contours in the velocity panels indicate the position of the dark bubble.

In the photospheric velocity maps (rows three and four), the granulation pattern is clearly visible throughout the temporal sequence. At 10:09:42 UT, a granule near the center of the FOV with an elongated shape shows more prominent blueshifts of -1.8 and $-1.3 \mathrm{~km} \mathrm{~s}^{-1}$ at the $80 \%$ and $10 \%$ intensity levels, respectively. One minute later, at 10:10:53 UT, the blueshifts have increased to -2.4 and $-2.0 \mathrm{~km} \mathrm{~s}^{-1}$, coinciding with the stronger linear polarization patch. At 10:12:01 UT, the LOS velocities start to decrease down to the -1.8 and $-1.1 \mathrm{~km} \mathrm{~s}^{-1}$ attained at 10:13:14 UT. Thus, the blueshifts increase their value 


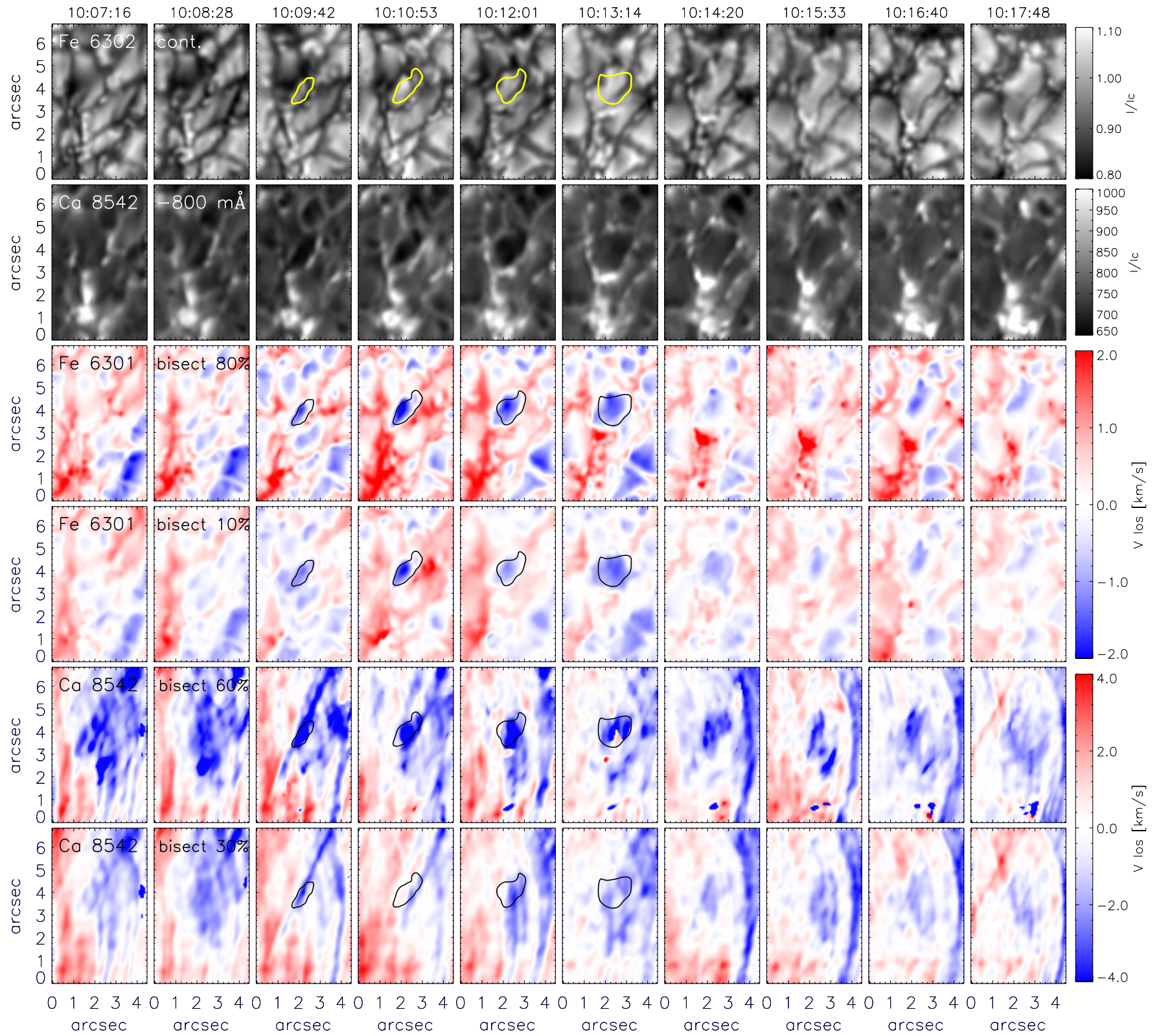

Figure 11. Temporal sequence of intensities and LOS velocities for flux emergence case 1, covering the interval from 10:07:16 to 10:17:48 UT. First and second rows: continuum intensity at $630.32 \mathrm{~nm}$ and Ca II $854.2-0.08 \mathrm{~nm}$ intensity filtergrams. Third and fourth rows: Fe I $630.15 \mathrm{~nm}$ bisector velocities computed at the $80 \%$ and $10 \%$ intensity levels. Fifth and sixth rows: chromospheric velocities determined from Ca II $854.2 \mathrm{~nm}$ line bisectors at the $60 \%$ and $30 \%$ intensity levels. Velocities are clipped at $\pm 2 \mathrm{~km} \mathrm{~s}^{-1}$ in the photosphere and $\pm 4 \mathrm{~km} \mathrm{~s}^{-1}$ in the chromosphere. Note the strong redshifts of $2 \mathrm{~km} \mathrm{~s}^{-1}$ that appear in coincidence with the negative polarity foot, particularly at the $80 \%$ intensity level around $(x, y)=\left(1^{\prime \prime}-2^{\prime \prime}, 2^{\prime \prime}-3^{\prime \prime}\right)$. Brigthenings also occur at the same position. The contours indicate the presence of the dark bubble during its life.

(A color version of this figure is available in the online journal.)

at the beginning, peak at 10:10:53 UT (regardless of the bisector level), and decrease in the next line scans. The bisector closer to the line core reveals smaller Doppler shifts than the bisector closer to the continuum.

Redshifts are also observed at the feet of the bubble in the photospheric velocity panels. Their average value is $2 \mathrm{~km} \mathrm{~s}^{-1}$ as measured at the $80 \%$ intensity level. Peaks of $3 \mathrm{~km} \mathrm{~s}^{-1}$ are reached at 10:10:53 and 10:14:20 UT, while velocities of $3.7 \mathrm{~km} \mathrm{~s}^{-1}$ are detected at 10:15:33 UT. These strong downward flows are probably the result of drainage of material along the legs of the rising magnetic bubble. They coincide with strong brightenings in the intensity filtergrams around $(x, y)=$ $\left(1^{\prime \prime}, 0^{\prime \prime}-3^{\prime \prime}\right)$ and $\left(2^{\prime \prime}, 2^{\prime \prime}-3^{\prime \prime}\right)$. Interestingly, those locations are associated with the negative polarity leg and present a strong magnetic field. The $10 \%$ bisector level also shows redshifts at the same positions but of lesser magnitude.

The last two rows of Figure 11 display chromospheric velocities as measured by the Ca II $854.2 \mathrm{~nm}$ bisectors. At the beginning of the temporal sequence, most of the FOV is dominated by strong blueshifts associated with chromospheric fibrils that are opaque and do not permit the detection of features below them. At 10:09:42 UT, a narrow fibril seems to cross over the dark bubble, with an average velocity of $-5.0 \mathrm{~km} \mathrm{~s}^{-1}$. In the next time step, the fibril has mostly disappeared, and the $60 \%$ bisector velocity shows blueshifts of $-5.5 \mathrm{~km} \mathrm{~s}^{-1}$ at the position of the dark bubble. The bubble is well detected 
until the end of the sequence when the velocities have slowed down to $-1 \mathrm{~km} \mathrm{~s}^{-1}$. The $30 \%$ bisector velocity indicates weaker upward motions in the dark bubble with signals that are delayed with respect to the $60 \%$ bisector velocity. At those heights, the fibrils are detected until 10:10:53 UT, giving way to the bubble at 10:12:01 UT with blueshifts of $-3 \mathrm{~km} \mathrm{~s}^{-1}$. The velocity slows down to $-1 \mathrm{~km} \mathrm{~s}^{-1}$ at the end of the sequence.

As mentioned before, there is the possibility that some of the blueshifts inferred from the Ca II $854.2 \mathrm{~nm}$ line are not real Doppler velocities but an artifact of the emission peak that occurs in the red wing of the line at some locations. However, most of the bubble interior does not show profiles in emission (see the blue contours in Figure 13), so a large fraction of the blueshifts detected in the chromosphere must be legitimate. This issue will be examined in more detail in Paper II of this series.

\subsection{Magnetic Properties}

\subsubsection{Photospheric Fields}

From top to bottom, Figure 12 presents the temporal evolution of the Ca II $854.2-0.08 \mathrm{~nm}$ filtergrams, together with the field strength, field inclination, LOS velocity, and temperature at $\log \tau=0$ and $\log \tau=-2$ inferred from the inversion of the Stokes profiles of the two Fe I lines for flux emergence event 1 . The appearance of the positive leg is well visible at 10:09:42 UT in the field strength map and one minute later in the inclination map. It then builds up. The newly formed positive leg contains field strengths that vary between 300 and $500 \mathrm{G}$ from its appearance at 10:09:42 UT until the end of the sequence. Inclinations change from $86^{\circ}$ when the leg appears to $50^{\circ}$ at the end of the sequence. The negative leg yields stronger fields, ranging from 400 to $800 \mathrm{G}$, and inclinations varying between $145^{\circ}$ and $110^{\circ}$. The pattern of the field strength at the center of the magnetic bubble follows that shown in Figure 7 by the linear polarization patch. At 10:09:42 UT, the center of the bubble presents a magnetic strength of $300 \mathrm{G}$ that grows up to $480 \mathrm{G}$ in the next time step and then decreases to 370,200 , and finally $120 \mathrm{G}$ at 10:14:20 UT. The inclinations at the center of the bubble lie between $75^{\circ}$ and $95^{\circ}$. Thus, a horizontal field traverses the photosphere being particularly strong at 10:10:53 and 10:12:01 UT, in agreement with Figure 7.

The velocities show a normal granulation pattern in the first frames, but later on, the granule at the center of the FOV stands out among the others with increased upward velocities that reach a peak of $-3 \mathrm{~km} \mathrm{~s}^{-1}$ at 10:10:53 UT before slowing down to $-1 \mathrm{~km} \mathrm{~s}^{-1}$ at 10:15:33 UT. The photospheric temperature at $\log \tau=0$ does not show any trace of the dark bubble, in agreement with the observed continuum intensity pattern. However, the dark bubble is easily identified in the temperature map at $\log \tau=-2$, where it shows a time-averaged deficit of $150 \mathrm{~K}$ with respect to the mean temperature of $5020 \mathrm{~K}$. At 10:13:14 UT, the deficit reaches its maximum value of $250 \mathrm{~K}$. Note that the dark bubble seen in the temperature maps cannot be mistaken with reverse granulation, since its size is considerably larger than that of the underlying granule. This means that the magnetic bubble is cooler than the surroundings in the mid photosphere but not below it (we recall that $\log \tau=0$ refers to the continuum forming region and $\log \tau=-2$ to a layer some $300 \mathrm{~km}$ above it).

\subsubsection{Chromospheric Fields}

The chromospheric magnetic signals are very small and thus difficult to measure. We do not observe Stokes $Q$ or $U$ signals in the Ca II $854.2 \mathrm{~nm}$ line above the noise level. However, we do detect Stokes $V$ signals that allow us to estimate the longitudinal field in chromospheric layers. We have utilized the weak field approximation for this purpose, as described in Section 2.3.

Figure 13 shows intensity filtergrams in the low and mid photosphere (first two panels), together with longitudinal magnetic field maps in the low photosphere (panel 3), the mid photosphere (panel 4), and the mid chromosphere (panel 5). The longitudinal field map displayed in panel 3 has been constructed using the inversion results as $B_{\mathrm{LOS}}=B \cos \gamma$. The longitudinal fields derived from the $\mathrm{Ca}$ II line have been obtained by applying the weak field approximation to the line wings (excluding the central $\pm 300 \mathrm{~m} \AA$; panel 4) and to the region within the profile "knees" $( \pm 300 \mathrm{~m} \AA$ around the line core; panel 5) in order to separate the photospheric contribution from the purely chromospheric contribution.

In Figure 13, the red contours outline pixels with chromospheric longitudinal fields stronger than $\phi=-90 \mathrm{G}$ (three times the noise level). As can be seen, the most intense chromospheric fields occur at the position of the lower half-moonshaped magnetic leg. The pixel marked with a cross in panel 2 at $(x, y)=\left(2^{\prime \prime}, 2^{\prime \prime} .5\right)$, for example, has a field strength of $750 \mathrm{G}$ and an inclination of $123^{\circ}$ in the photosphere, i.e., a longitudinal field of $-410 \mathrm{G}$, but only $-175 \mathrm{G}$ in the mid chromosphere as inferred from the Ca II $854.2 \mathrm{~nm}$ line core. This change by a factor of 2.3 between the two layers sampled by the corresponding maps implies a rapid decrease of the field strength with height, or a progressively larger inclination of the field toward higher layers, or both.

The polarity of the magnetic feet is the same-negative-in the photosphere and the chromosphere. But that does not always seem to hold for pixels in the vicinity of the magnetic feet, as some of them show opposite-polarity (positive) $V$ profiles in the chromosphere. We have pinpointed those pixels with blue contours in the second panel of Figure 13. Whenever there is a change in the sign of Stokes $V$ between the photosphere and the chromosphere, the Ca II $854.2 \mathrm{~nm}$ Stokes I profiles show emission instead of absorption. This reversal of the polarity is only apparent: in the weak field regime, the Stokes $V$ sign is determined by the sign of $\delta I / \delta \lambda$. When the line goes in emission, $\delta I / \delta \lambda$ changes sign and so does Stokes $V$. Thus, also for those pixels the magnetic polarity is the same in the two atmospheric layers. We have previously observed this behavior in the Stokes $I$ and $V$ profiles of Figure 10, which switch from absorption to an asymmetric emission peak in the red wing. Other examples of apparent polarity reversals in chromospheric lines have been presented (and explained) by Martínez Pillet et al. (1990), Sánchez Almeida (1997), and de la Cruz Rodríguez et al. (2013).

The blue contours show that the magnetic bubble is surrounded by pixels with chromospheric Stokes I profiles partially in emission, which is indicative of plasma being displaced at a different speed and possibly with much higher temperature in some layers along the LOS. Whether such higher temperatures would be achieved by reconnection or by some other heating mechanism remains to be determined.

\section{NUMERICAL SIMULATIONS}

Further insight into the observed phenomena may be gained with the help of "realistic" numerical simulations. In this case, the term "realistic" means models in which enough physical mechanisms are included so that observables synthesized from the simulations can be directly compared with our observations. 

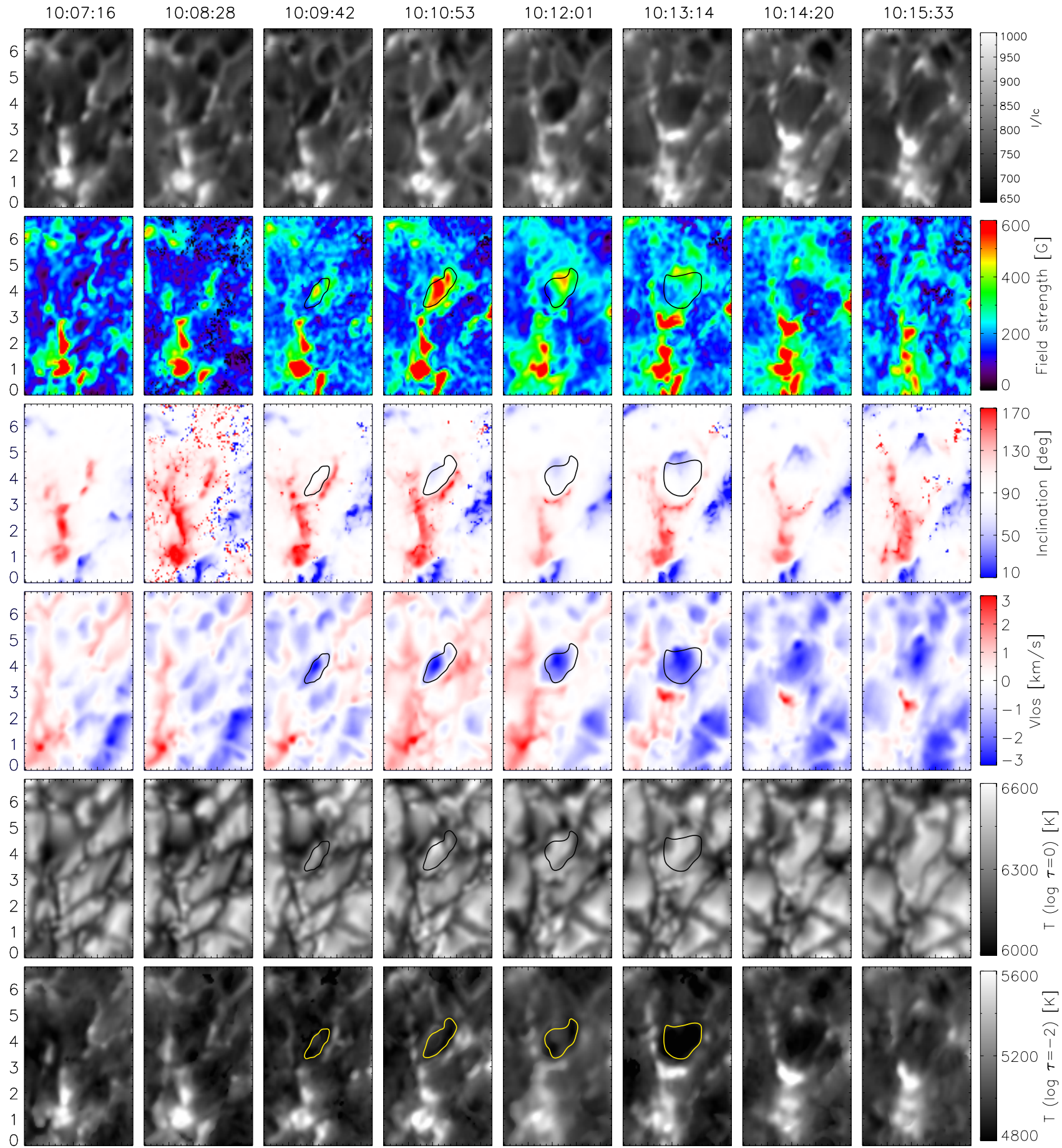

Figure 12. Temporal evolution of the atmospheric parameters inferred from the inversion of the Fe $\mathrm{I} 630 \mathrm{~nm}$ lines for flux emergence event 1 . From top to bottom: Ca II $854.2 \mathrm{~nm}-0.08$ filtergram, field strength, inclination of the vector magnetic field, LOS velocity, temperature at $\log \tau=0$, and temperature at $\log \tau=-2$. The sequence covers the interval from 10:07:16 to 10:15:33 UT. The contours outline the position of the dark bubble.

(A color version of this figure is available in the online journal.)

We have run an experiment in which a magnetic flux sheet that is injected into the convection zone rises to the photosphere and later breaks through into the chromosphere in much the same way as we believe is happening in the observations described above.

The models presented here are performed on a $24 \times 24 \times$ $16.5 \mathrm{Mm}^{3}$ cube discretized on a grid containing $504 \times 504 \times 496$ cells, with a horizontal grid size of $41 \mathrm{~km}$ and a variable spacing in the vertical direction ranging from $20 \mathrm{~km}$ in the photosphere, gradually increasing to $95 \mathrm{~km}$ in the corona $14 \mathrm{Mm}$ above the photosphere, to $85 \mathrm{~km}$ in the convection zone $2.5 \mathrm{Mm}$ below the photosphere. The model is calculated using the Bifrost code (Gudiksen et al. 2011) and includes an equation of state based on solar abundances in LTE, optically thick radiative transfer and losses from the photosphere and chromosphere including scattering (Skartlien 2000; Hayek et al. 2010). In the middle and 


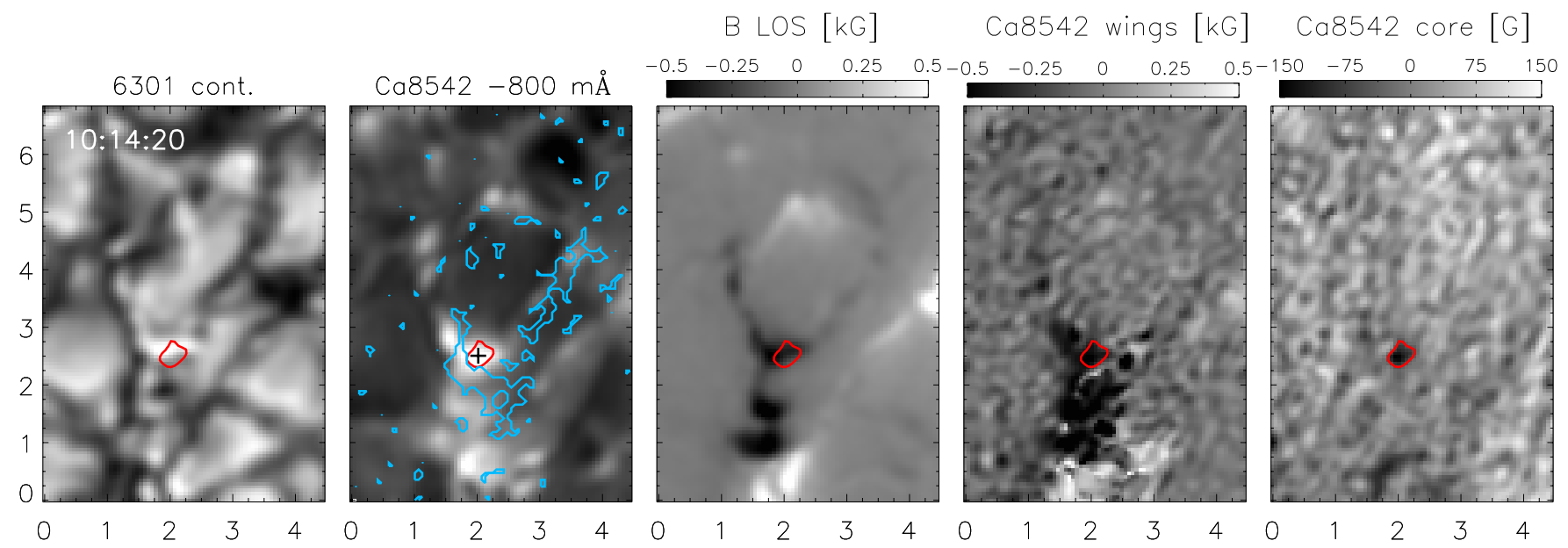

Figure 13. From left to right: Stokes $I$ map in the continuum at $630.32 \mathrm{~nm}$, Ca II $854.2-0.08 \mathrm{~nm}$ filtergrams, longitudinal photospheric magnetic field from the inversion of the two Fe I lines, and longitudinal magnetic field from the wings and the core of the Ca II $854.2 \mathrm{~nm}$ line for flux emergence event 1 . All the maps correspond to 10:14:20 UT. The red contours highlight regions with chromospheric longitudinal fields stronger than $-90 \mathrm{G}$ and have been superimposed onto the other four panels. The cross in the second panel marks a pixel in the magnetic legs, which is discussed in the text. The blue contours in the second panel outline pixels with intensity profiles in emission.

(A color version of this figure is available in the online journal.)

upper chromosphere as well as in the transition region, a recipe for effectively thin radiation is used, as described by Carlsson \& Leenaarts (2012). In the corona, thermal conduction along the magnetic field plays an important role; this is treated by operator splitting where the resulting implicit operator for conduction is solved using a multi-grid method as described in Gudiksen et al. (2011).

In the simulation, a magnetic sheet aligned with the $y$-axis, stretching from $x=4 \mathrm{Mm}$ to $x=16 \mathrm{Mm}$, and with a strength of $B_{y}=3363 \mathrm{G}$ is inserted at the bottom boundary. This particular field strength has been chosen after experimenting with weaker flux sheets that failed to produce the desired emergence. The insertion continues for $1 \mathrm{hr} 45$ minutes $(6310 \mathrm{~s})$ before it is turned off. The magnetic field of the sheet reaches the photosphere fairly rapidly (within an hour or so), but at the photosphere it is no longer carried by convection nor sufficiently buoyant and therefore becomes "stuck," slowly gaining strength as the field from below piles up.

In Figure 14 we show a vertical cut of the evolution of the magnetic field strength, density, vertical velocity, and temperature along with the emergent intensity from the photosphere and the lower chromosphere (i.e., similar to the emergent intensity in the white light continuum and near the line core of a strong chromospheric line such as Ca II $854.2 \mathrm{~nm}$ ) at four different times during the simulation. Initially, the emerging magnetic field has only reached to some $1.5 \mathrm{Mm}$ below the photosphere, and the photosphere and chromosphere are dominated by granular flows and chromospheric oscillations. At $t=1^{\mathrm{h}} 59^{\mathrm{m}} 39^{\mathrm{s}}$, the field fills the convection zone up to the photosphere but has not yet penetrated the upper photosphere nor the chromosphere. This happens first two minutes later, when the field gradient in the vertical direction becomes strong enough to trigger the magnetic buoyancy instability (Parker instability): when the magnetic field strength decreases with height, it reduces the gas pressure gradient and sets up a situation where heavier fluid overlies a lighter fluid as described by Archontis et al. (2004) and references therein. At this point, the field breaks through the photosphere, and a bubble of high field strength emerges into the chromosphere and corona. This bubble expands rapidly and adiabatic cooling ensues; the chromospheric material becomes very cool as the bubble expands far beyond the original dimensions of the flux emerging into the photosphere. Though the event shown here is too weak to significantly perturb the granulation pattern in the photosphere, we see that as the bubble rises, the chromospheric emission becomes very low where the temperatures are lowest, while enhanced emission occurs near the edges. Upon expanding into the chromosphere, the bubble achieves a nearly spherical shape as can be seen in the $3 \mathrm{D}$ rendering of Figure 15. At this stage, it has a diameter of some $3 \mathrm{Mm}$.

The response of the simulated photosphere and chromosphere to the expansion of the emerging magnetic flux is further shown in Figure 16. The evolution of the photospheric continuum intensity and magnetic field are plotted alongside the temperature at five separate heights $(200,350,500,700,1000 \mathrm{~km})$ in the lower and middle chromosphere and the vertical velocity at two heights $(200,1000 \mathrm{~km})$. Initially, a small region of strong magnetic field (both vertical and horizontal) pierces the photosphere. At this time, there is no response in the temperature nor in the velocity pattern at higher altitudes. One minute later, at 1:53:19, a circular region of slightly cooler gas appears over the emerging flux region $350 \mathrm{~km}$ above the continuum forming layer. (Note that we do not see any obvious response in the temperature at a height of $200 \mathrm{~km}$ at that time nor indeed at any later time.) This structure is similar to that appearing in the inverted temperature maps at $\log \tau=-2$, which show the dark bubble in the middle photosphere. The cool region at $350 \mathrm{~km}$ grows larger as time progresses and at 2:01:19 has a diameter of roughly $3^{\prime \prime}$. This temperature response is mirrored at greater heights, but the cooling region appears later, at 1:54:19 at $700 \mathrm{~km}$ and at 1:55:19 at $1000 \mathrm{~km}$, implying an ascent speed of $4.2 \mathrm{~km} \mathrm{~s}^{-1}$. At these great heights, the temperature becomes very low in the "bubble," falling to less than $2000 \mathrm{~K}$. Though the temperature response is very weak or missing at $200 \mathrm{~km}$, the flux emergence is quite evident in the velocity signal, with upflows of order $3 \mathrm{~km} \mathrm{~s}^{-1}$ outlining the cool bubble above. At the edges of the granule, and especially centered on the regions of greater vertical field strength, there are downflows of $3 \mathrm{~km} \mathrm{~s}^{-1}$. At $1000 \mathrm{~km}$, where 


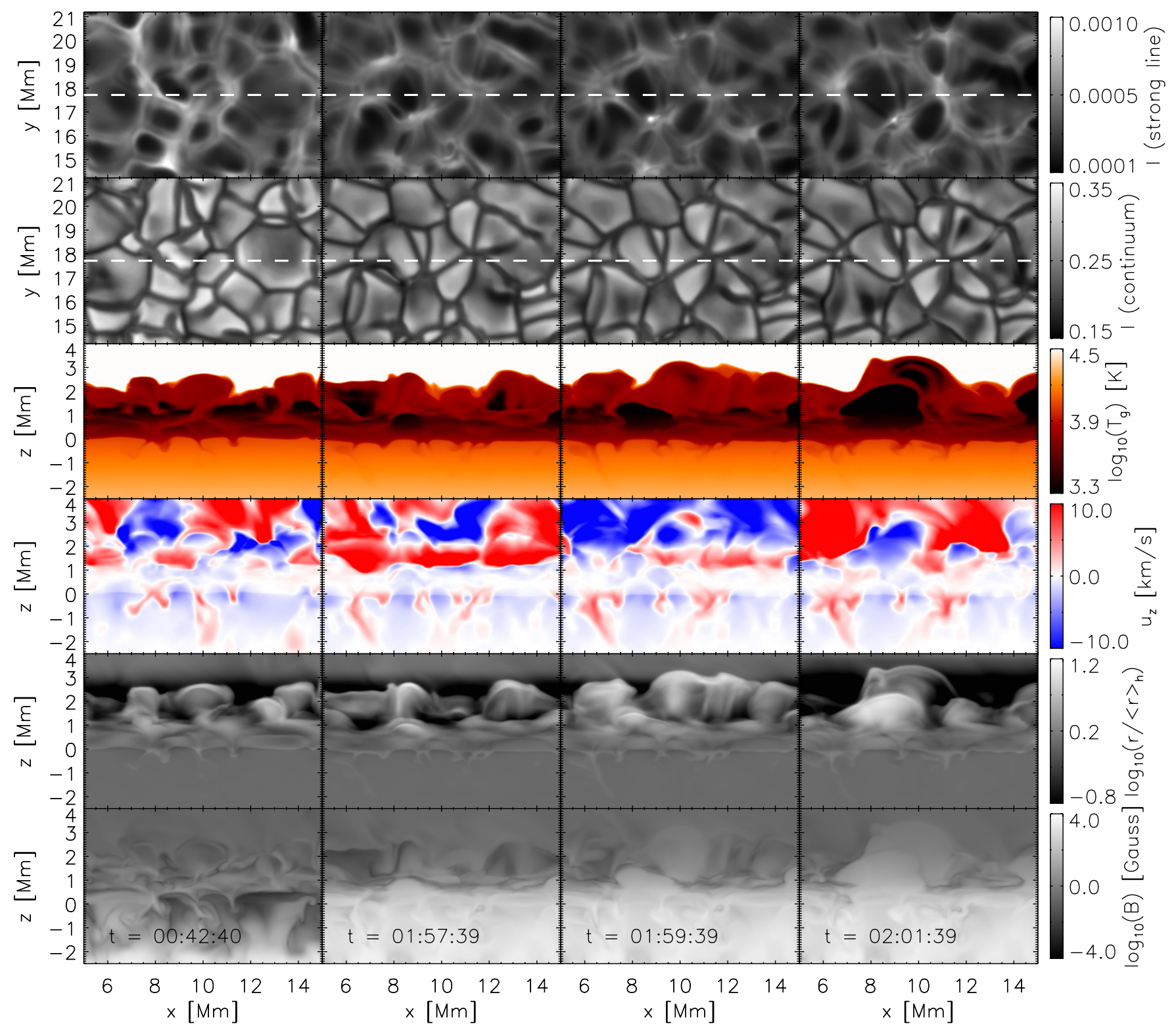

Figure 14. Views of the simulation box at four different times. Top two rows: intensities in the horizontal plane that would be observed in the core of a strong chromospheric line (such as Ca II $854.2 \mathrm{~nm}$ ) and in the continuum at $630 \mathrm{~nm}$. Bottom four rows: vertical cuts showing temperature, vertical velocity, gas density, and magnetic field strength. The dashed lines in the top two rows indicate the position of the selected vertical slices.

(A color version of this figure is available in the online journal.)

the bubble is very pronounced in temperature, we find upflows of some $10 \mathrm{~km} \mathrm{~s}^{-1}$ surrounded by downflows with approximately the same amplitude.

In the photosphere, the horizontal magnetic field strength of the rising flux is of the order several hundred $\mathrm{G}$, increasing to $1 \mathrm{kG}$ near the edges of the granule containing the emerging field. The vertical field is much more concentrated to the intergranular lanes and reaches a maximum of some $1150 \mathrm{G}$. At a height of $700 \mathrm{~km}$, we find that as the magnetic bubble rises and expands, the horizontal magnetic field grows to $B_{\mathrm{h}}=100 \mathrm{G}$ and is concentrated toward the center of the bubble, and while the vertical field grows to a similar amplitude, it is concentrated to the edges.

The evolution of the simulated dark bubble is, with the exception of perturbed granulation, remarkably similar to what is observed. This includes the field strength and LOS velocities of the emerging flux in the photosphere and the chromosphere, the ascent speed and expansion velocity of the bubble, its temperature deficit in the middle photosphere, and the duration of the entire event. In the model, the emerging field continues to expand and eventually, after a series of reconnection events, merges and/or replaces the ambient coronal field.

\section{DISCUSSION AND CONCLUSIONS}

With the advent of very high resolution spectropolarimetric measurements, our understanding of small-scale flux emergence in the solar atmosphere has improved dramatically. So has the realism of numerical simulations of this phenomenon, thanks to ever-increasing computer capabilities. Both within active regions and in the quiet Sun, a common form of flux emergence is the rise of simple $\Omega$-shaped magnetic loops that intersect the photosphere at two magnetic footpoints. The present paper deals with a more complex kind of flux emergence, a 3D semi-sphere 


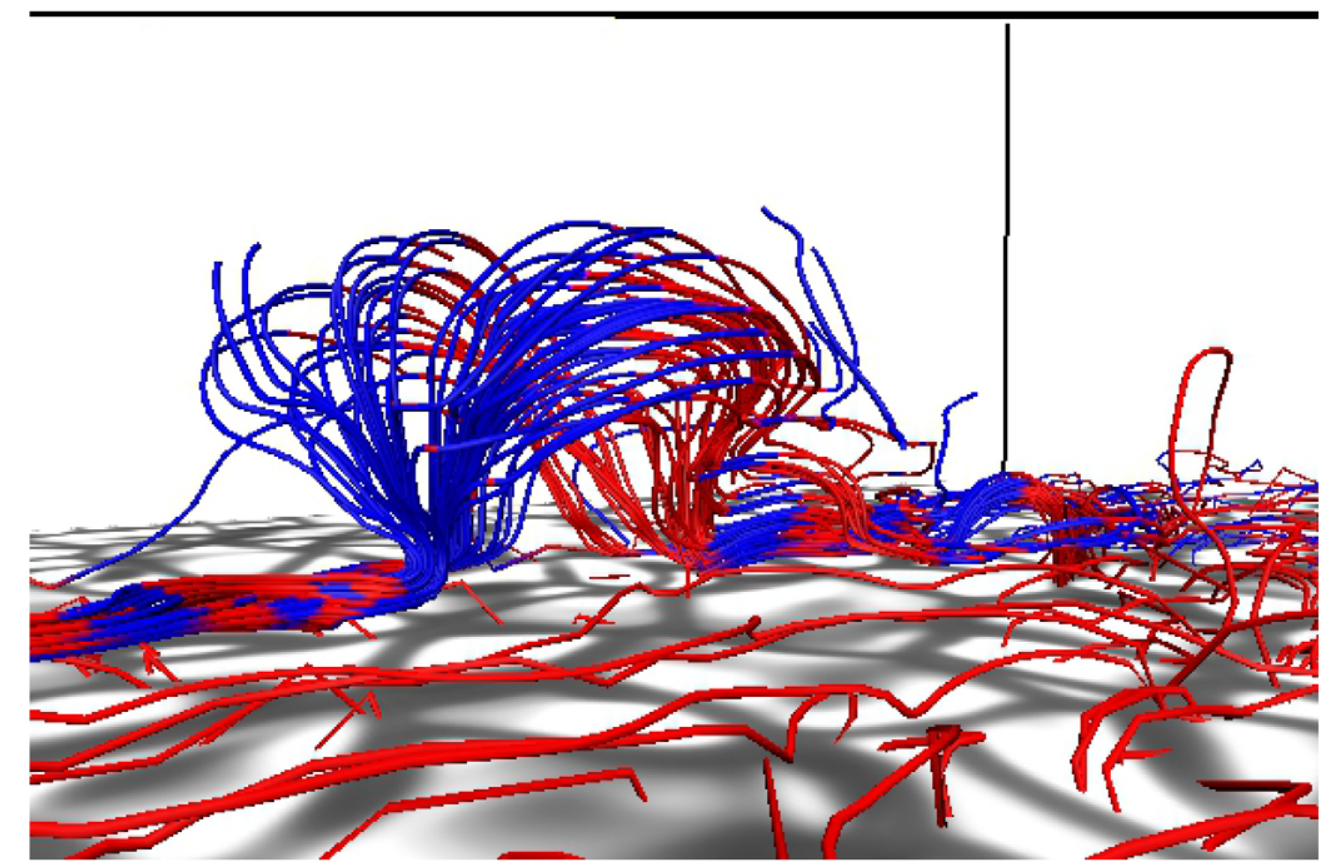

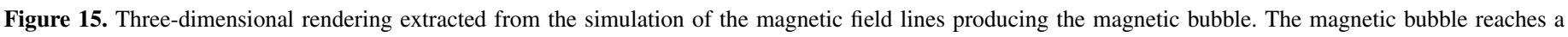
diameter of $3 \mathrm{Mm}$. Red and blue lines indicate fields pointing upward and downward, respectively.

(A color version of this figure is available in the online journal.)

that we have called magnetic bubble. Such a bubble pierces the photosphere, producing half-moon shaped magnetic feet. To our knowledge, only Guglielmino et al. (2012) have observed flux emergence events with similar crescent-shaped magnetic legs. Here we present multiwavelength observations and simulations that allow us to get a better understanding of the emergence of these magnetic features.

We have observed some of the typical signatures of flux emergence: abnormal granulation, separation of magnetic feet of opposite polarity, and brightenings in chromospheric layers. We have quantified how the magnetic bubble expands horizontally and rises vertically. The expansion and rise seem to happen in an intermittent way rather than in a continuous and smooth fashion. But the most remarkable observational feature-and the main result of this paper-is the appearance of a dark bubble contained within the magnetic enclosure in the mid-photosphere and above. This bubble is magnetized and pumps magnetic flux into the chromosphere. Surrounding the bubble we have detected Ca II $854.2 \mathrm{~nm}$ intensity profiles with an emission peak in their red wings. In order to produce such a peak, a very high velocity gradient is needed along the LOS, possibly coupled with strong temperature enhancements at some height in the atmosphere. These are perhaps the signs of reconnection happening at the edges of the bubble or of other heating mechanisms.

We have measured the distance between the magnetic feet and found values of up to 6".6, with horizontal separation velocities of about $4 \mathrm{~km} \mathrm{~s}^{-1}$ on average. Otsuji et al. (2007) reported separation speeds of $4.2 \mathrm{~km} \mathrm{~s}^{-1}$ during the initial phases of flux emergence and some $1 \mathrm{~km} \mathrm{~s}^{-1}$ later on. The dark bubble shows maximum sizes of $3^{\prime \prime} \times 1^{\prime \prime}$ and horizontal expansion velocities similar to those of the magnetic feet.

The rising gas has LOS velocities of around $-2.5 \mathrm{~km} \mathrm{~s}^{-1}$ in the photosphere and $-3 \mathrm{~km} \mathrm{~s}^{-1}$ in the chromosphere. Strong downflows are detected at the position of the magnetic feet, with peaks of $4 \mathrm{~km} \mathrm{~s}^{-1}$. Observational studies like Guglielmino et al. (2008) and Guglielmino et al. (2012) report slightly smaller values of -1 to $-2 \mathrm{~km} \mathrm{~s}^{-1}$ for the upflows and 1 to $2 \mathrm{~km} \mathrm{~s}^{-1}$ for the downflows. Numerical simulations by Cheung et al. (2008) yield upward velocities as small as $-0.5 \mathrm{~km} \mathrm{~s}^{-1}$. However, the simulations of Martínez-Sykora et al. (2008) give photospheric values of $-2.5 \mathrm{~km} \mathrm{~s}^{-1}$ for the upflows and $3-5 \mathrm{~km} \mathrm{~s}^{-1}$ for the downflows. In the chromosphere, they find $-7 \mathrm{~km} \mathrm{~s}^{-1}$ and $10 \mathrm{~km} \mathrm{~s}^{-1}$, respectively. These values are similar to ours.

Brightenings, in particular where the new flux system interacts with the preexisting ambient field, are common in emergence events as reported by, e.g., Guglielmino et al. (2008) and Vargas Domínguez et al. (2012). We also observed them. Figure 7 shows that brightenings are seen all across the Ca II $854.2 \mathrm{~nm}$ line profile, resembling the shape and position of the magnetic feet.

We have derived the magnetic properties of the emerging flux in the photosphere and the chromosphere. The longitudinal magnetic field at the position of the half-moon shaped magnetic legs is around $410 \mathrm{G}$ in the photosphere (corresponding to a field strength of $750 \mathrm{G}$ ) and $175 \mathrm{G}$ at the height of formation of the Ca II $854.2 \mathrm{~nm}$ line core, i.e., the middle chromosphere. The field strength in between the two magnetic feet is around 300 to $400 \mathrm{G}$ as determined from the inversion of the observed Fe I $630 \mathrm{~nm}$ lines. Guglielmino et al. (2012) also obtained maximum strengths of $400 \mathrm{G}$ in the photosphere with the IMAX instrument on board the SUNRISE balloon (Martínez Pillet et al. 2011). The numerical simulations performed here yield $\mathrm{hG}$ strengths for the horizontal field of the bubble interior and $1 \mathrm{kG}$ for the vertical field of the feet, $200 \mathrm{~km}$ above the continuum forming layer. At a height of $700 \mathrm{~km}$, the horizontal magnetic field has a strength of some $100 \mathrm{G}$ and is concentrated toward the center of the bubble, whereas the vertical field has similar strengths but is mainly seen near the magnetic legs. This is in excellent agreement with Cheung et al. (2008) and TortosaAndreu \& Moreno-Insertis (2009), who found $B_{\mathrm{h}}=100 \mathrm{G}$ at a height of $1000 \mathrm{~km}$. Martínez-Sykora et al. (2008) also obtained $80 \mathrm{G}$ at $900 \mathrm{~km}$. 


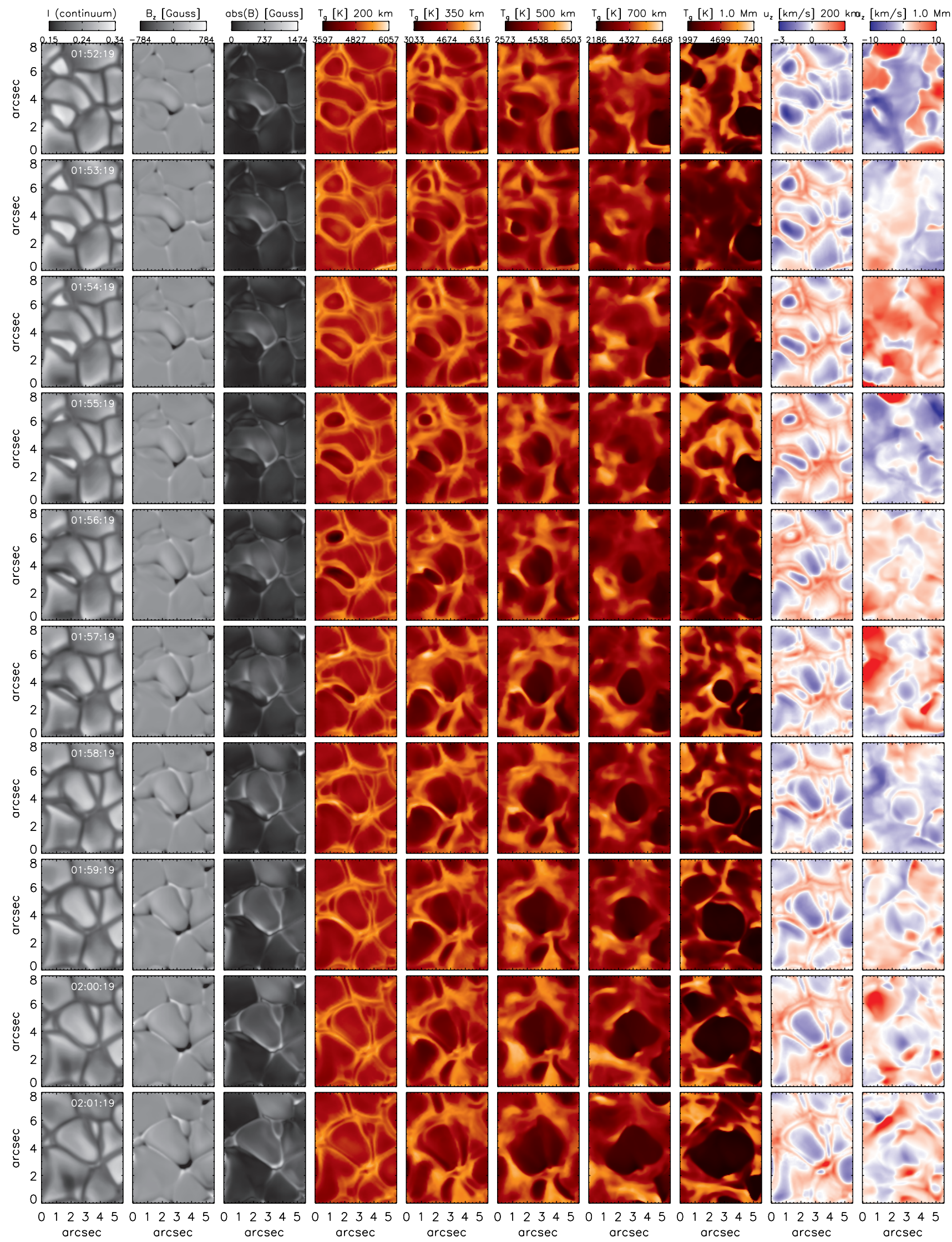

Figure 16. Temporal evolution between 1:52:19 and 2:01:19 simulation times of the white light intensity, vertical magnetic field, total magnetic field, and temperatures at $200,350,500,700$, and $1000 \mathrm{~km}$ above the photosphere, as well as the vertical velocity at 200 and $1000 \mathrm{~km}$ above the photosphere. The color scale for $B_{z}$ is set to $[-784,784] \mathrm{G}$, while for $|B|$ it is set to $[0,1474] \mathrm{G}$. The temperature scale ranges between 3597 and $6057 \mathrm{~K}$ at $200 \mathrm{~km}$, between 3033 and $6316 \mathrm{~K}$ at $350 \mathrm{~km}$, between 2573 and $6503 \mathrm{~K}$ at $500 \mathrm{~km}$, between 2186 and $6468 \mathrm{~K}$ at $700 \mathrm{~km}$, and between 1997 and $7401 \mathrm{~K}$ at $1000 \mathrm{~km}$. The velocity scale at $200 \mathrm{~km}$ is set to [-3,3] km s and at $1000 \mathrm{~km}$ is set to $[-10,10] \mathrm{km} \mathrm{s}^{-1}$ (red color represents downflows).

(A color version of this figure is available in the online journal.) 
Our realistic numerical simulations are able to reproduce, both qualitatively and quantitatively, the main observational results. This includes the field strength and the LOS velocities of the emerging flux, both in the photosphere and in the chromosphere, the expansion and rise of the bubble, the lower gas temperature of the bubble in the mid and high photosphere, and the total lifetime. The organization of the field lines in the simulation is shown in Figure 15. As can be seen, this is a truly spherical bubble that is expanding into the chromosphere after rising through the photosphere. Except at the position of the bubble, the photospheric field is essentially horizontal. The picture that can be drawn from both observations and simulations is one of an extended sheet of horizontal fields initially located in subphotospheric layers that rise into the atmosphere with the help of the vertical upflows of a granule. During the ascent, the fields adopt the form of a bubble and create a temperature deficit that is observed as a dark feature in the middle photosphere $(\log \tau=-2)$ and above.

Martínez-Sykora et al. (2008) and Tortosa-Andreu \& Moreno-Insertis (2009) detected similar dark bubbles in their simulations. The bubbles are formed by adiabatic cooling of flux emerging and expanding rapidly. Both papers quote temperatures of less than $3000 \mathrm{~K}$ for the dark bubbles at heights of 700 to $900 \mathrm{~km}$.

Using Hinode measurements, Vargas Domínguez et al. (2012) reported the existence of small-scale (2-4 Mm), short-lived (12 minutes) dark areas in a flux emergence event similar to the ones analyzed here. These features were observed in $\mathrm{Ca}$ II $\mathrm{H}$ filtergrams but also in photospheric $\mathrm{Na}$ I $589.6 \mathrm{~nm}$ Stokes I images. According to Vargas Domínguez et al. (2012), they appear in regions with negligible longitudinal magnetic field. Even though there are some differences (our bubbles present both horizontal and vertical fields and occur above $\log \tau=-2$ ), their observations may have targeted the same features at less spatial resolution and only with partial magnetic field information.

In Paper II of this series, we will invert the observed Ca II $854.2 \mathrm{~nm}$ profiles under non-LTE conditions to determine the properties of the magnetic bubble in the chromosphere, including the stratification of the gas temperature and the LOS velocity. We will investigate the origin of the emission features detected in the Ca II $854.2 \mathrm{~nm}$ red line wing and the possibility that they signal the presence of strong temperature enhancements at some height in the atmosphere. Also, we will compute synthetic Fe I $630 \mathrm{~nm}$ and Ca II $854.2 \mathrm{~nm}$ Stokes profiles from the simulations in order to compare them with the observed ones. Differences between the synthetic and observed profiles will provide additional constraints to our numerical model of flux emergence.

Part of the work presented here was done while A.O. was a Visiting Scientist at the Instituto de Astrofísica de Andalucía (CSIC). We gratefully acknowledge financial support by the Spanish MINECO through project AYA2012-39636-C06-05, including a percentage from European FEDER funds, by the Research Council of Norway through grants 208027/F50 and "Solar Atmospheric Modelling," by the European Research Council under the European Union's Seventh Framework Programme (FP7/2007-2013)/ERC Grant agreement No. 291058, and by the Programme for Supercomputing of the Research Council of Norway through grants of computing time. The Swedish $1 \mathrm{~m}$ Solar Telescope is operated by the Institute for Solar Physics of Stockholm University in the Spanish Observatorio del Roque de los Muchachos of the Instituto de Astrofísica de Canarias. This research has made use of NASA's Astrophysical Data System.

\section{REFERENCES}

Archontis, V., Moreno-Insertis, F., Galsgaard, K., Hood, A., \& O'Shea, E. 2004, A\&A, 426, 1047

Bellot Rubio, L. R., \& Orozco Suárez, D. 2012, ApJ, 757, 19

Caligari, P., Moreno-Insertis, F., \& Schussler, M. 1995, ApJ, 441, 886

Carlsson, M., \& Leenaarts, J. 2012, A\&A, 539, A39

Centeno, R., Socas-Navarro, H., Lites, B., et al. 2007, ApJL, 666, L137

Cheung, M. C. M., Schüssler, M., \& Moreno-Insertis, F. 2007, A\&A, 467, 703

Cheung, M. C. M., Schüssler, M., Tarbell, T. D., \& Title, A. M. 2008, ApJ, 687,1373

de la Cruz Rodríguez, J. 2010, PhD thesis, Stockholm Univ.

de la Cruz Rodríguez, J., Rouppe van der Voort, L., Socas-Navarro, H., \& van Noort, M. 2013, A\&A, 556, A115

De Pontieu, B. 2002, ApJ, 569, 474

Fisher, G. H., Fan, Y., Longcope, D. W., Linton, M. G., \& Pevtsov, A. A. 2000, SoPh, 192, 119

Fontenla, J. M., Avrett, E. H., \& Loeser, R. 1993, ApJ, 406, 319

Georgoulis, M. K., Rust, D. M., Bernasconi, P. N., \& Schmieder, B. 2002, ApJ, 575,506

Gingerich, O., Noyes, R. W., Kalkofen, W., \& Cuny, Y. 1971, SoPh, 18, 347

Gömöry, P., Balthasar, H., \& Puschmann, K. G. 2013, A\&A, 556, A7

Gömöry, P., Beck, C., Balthasar, H., et al. 2010, A\&A, 511, A14

Gudiksen, B. V., Carlsson, M., Hansteen, V. H., et al. 2011, A\&A, 531, A154

Guglielmino, S. L. 2012, in ASP Conf. Ser. 455, 4th Hinode Science Meeting: Unsolved Problems and Recent Insights, ed. L. R. Bellot Rubio, F. Reale, \& M. Carlsson (San Francisco, CA: ASP), 109

Guglielmino, S. L., Bellot Rubio, L. R., Zuccarello, F., et al. 2010, ApJ, 724, 1083

Guglielmino, S. L., Martínez Pillet, V., Bonet, J. A., et al. 2012, ApJ, 745,160

Guglielmino, S. L., Zuccarello, F., Romano, P., \& Bellot Rubio, L. R. 2008, ApJL, 688, L111

Hagenaar, H. J., Schrijver, C. J., \& Title, A. M. 2003, ApJ, 584, 1107

Hayek, W., Asplund, M., Carlsson, M., et al. 2010, A\&A, 517, A49

Ishikawa, R., \& Tsuneta, S. 2009, A\&A, 495, 607

Ishikawa, R., Tsuneta, S., Ichimoto, K., et al. 2008, A\&A, 481, L25

Jouve, L., \& Brun, A. S. 2009, ApJ, 701, 1300

Kosugi, T., Matsuzaki, K., Sakao, T., et al. 2007, SoPh, 243, 3

Landi degl'Innocenti, E., \& Landolfi, M. 2004, Polarization in Spectral Lines (Astrophysics and Space Library, Vol. 307; Dordrecht: Kluwer)

Leenaarts, J., Carlsson, M., Hansteen, V., \& Rouppe van der Voort, L. 2009, ApJL, 694, L128

Leenaarts, J., Rutten, R. J., Reardon, K., Carlsson, M., \& Hansteen, V. 2010, ApJ, 709,1362

Lites, B. W., Leka, K. D., Skumanich, A., Martinez Pillet, V., \& Shimizu, T. 1996, ApJ, 460, 1019

Martin, S. F., \& Harvey, K. L. 1979, SoPh, 64, 93

Martínez González, M. J., \& Bellot Rubio, L. R. 2009, ApJ, 700, 1391

Martínez González, M. J., Collados, M., Ruiz Cobo, B., \& Solanki, S. K. 2007, A\&A, 469, L39

Martínez González, M. J., Manso Sainz, R., Asensio Ramos, A., \& Bellot Rubio, L. R. 2010, ApJL, 714, L94

Martínez Pillet, V., Del Toro Iniesta, J. C., Álvarez-Herrero, A., et al. 2011, SoPh, 268, 57

Martínez Pillet, V., Garcia Lopez, R. J., del Toro Iniesta, J. C., et al. 1990, ApJL, 361, L81

Martínez-Sykora, J., Hansteen, V., \& Carlsson, M. 2008, ApJ, 679, 871

Moreno-Insertis, F. 2012, in ASP Conf. Ser. 455, 4th Hinode Science Meeting: Unsolved Problems and Recent Insights, ed. L. R. Bellot Rubio, F. Reale, \& M. Carlsson (San Francisco, CA: ASP), 91

Moreno-Insertis, F., \& Emonet, T. 1996, ApJL, 472, L53

Nelson, N. J., Brown, B. P., Brun, A. S., Miesch, M. S., \& Toomre, J. 2011, ApJL, 739, L38

Orozco Suárez, D., Bellot Rubio, L. R., del Toro Iniesta, J. C., \& Tsuneta, S. 2008, A\&A, 481, L33

Otsuji, K., Shibata, K., Kitai, R., et al. 2007, PASJ, 59, 649

Ruiz Cobo, B., \& del Toro Iniesta, J. C. 1992, ApJ, 398, 375

Sainz Dalda, A., Martínez-Sykora, J., Bellot Rubio, L., \& Title, A. 2012, ApJ, 748,38

Sánchez Almeida, J. 1997, A\&A, 324, 763 
Scharmer, G. B., Bjelksjo, K., Korhonen, T. K., Lindberg, B., \& Petterson, B. 2003, Proc. SPIE, 4853, 341

Scharmer, G. B., Narayan, G., Hillberg, T., et al. 2008, ApJL, 689, L69

Schlichenmaier, R., Rezaei, R., Bello González, N., \& Waldmann, T. A. 2010, A\&A, 512, L1

Schlichenmaier, R., Rezaei, R., \& González, N. B. 2012, in ASP Conf. Ser. 455, 4th Hinode Science Meeting: Unsolved Problems and Recent Insights, ed. L. R. Bellot Rubio, F. Reale, \& M. Carlsson (San Francisco, CA: ASP), 61 Selbing, J. 2005, MSc thesis, Stockholm Univ.

Shine, R. A., Title, A. M., Tarbell, T. D., et al. 1994, ApJ, 430, 413
Skartlien, R. 2000, ApJ, 536, 465

Tortosa-Andreu, A., \& Moreno-Insertis, F. 2009, A\&A, 507, 949

van Noort, M., Rouppe van der Voort, L., \& Löfdahl, M. G. 2005, SoPh, 228, 191

Vargas Domínguez, S., van Driel-Gesztelyi, L., \& Bellot Rubio, L. R. 2012, SoPh, 278, 99

Wang, J., Zhou, G., Jin, C., \& Li, H. 2012, SoPh, 278, 299

Watanabe, H., Vissers, G., Kitai, R., Rouppe van der Voort, L., \& Rutten, R. J. 2011, ApJ, 736, 71

Weber, M. A., Fan, Y., \& Miesch, M. S. 2011, ApJ, 741, 11 